\title{
AFFINE HECKE ALGEBRAS AND THEIR GRADED VERSION
}

\author{
GEORGE LUSZTIG
}

Dedicated to Sir Michael Atiyah on his sixtieth birthday

\section{INTRODUCTION}

0.1. Let $H_{v_{0}}$ be an affine Hecke algebra with parameter $v_{0} \in \mathbf{C}^{*}$ assumed to be of infinite order. (The basis elements $T_{s} \in H_{v_{0}}$ corresponding to simple reflections $s$ satisfy $\left(T_{s}+1\right)\left(T_{s}-v_{0}^{2 c(s)}\right)=0$, where $c(s) \in \mathbf{N}$ depend on $s$ and are subject only to $c(s)=c\left(s^{\prime}\right)$ whenever $s, s^{\prime}$ are conjugate in the affine Weyl group.) Such Hecke algebras appear naturally in the representation theory of semisimple $p$-adic groups, and understanding their representation theory is a question of considerable interest.

Consider the "special case" where $c(s)$ is independent of $s$ and the coroots generate a direct summand. In this "special case," the question above has been studied in [1] and a classification of the simple modules was obtained. The approach of [1] was based on equivariant $K$-theory.

This approach can be attempted in the general case (some indications are given in $[5,0.3]$ ), but there appear to be some serious difficulties in carrying it out.

0.2. On the other hand, in [5] we introduced some algebras $\bar{H}_{r_{0}}$, depending on a parameter $r_{0} \in \mathbf{C}$, which are graded analogues of $H_{v_{0}}$. The graded algebras $\bar{H}_{r_{0}}$ are in many respects simpler than $H_{v_{0}}$, and in [5] the representation theory of $\bar{H}_{r_{0}}$ is studied using equivariant homology. Moreover, we can make the machinery of intersection cohomology work for us in the study of $\bar{H}_{r_{0}}$, while in the $K$-theory context of $H_{v_{0}}$ it is not clear how to do this. In particular, the difficulties mentioned above disappear when $H_{v_{0}}$ is replaced by $\bar{H}_{r_{0}}$.

For this reason, it seemed desirable to try to connect the representation theories of $H_{v_{0}}$ and $\bar{H}_{r_{0}}$. In this paper, we shall prove that the classification of simple $H_{v_{0}}$-modules can be reduced to the same problem, where $H_{v_{0}}$ is replaced essentially by $\bar{H}_{r_{0}}$. This makes it possible to study the representation theory of $H_{v_{0}}$ without $K$-theory but with the aid of $\bar{H}_{r_{0}}$. (This is analogous to studying the representations of a Lie group using the theory of Lie algebras.) It should

Received by the editors March 14, 1989.

1980 Mathematics Subject Classification (1985 Revision). Primary 16A89, 16A03. 
be pointed out that this approach fails when $v_{0}$ is a root of 1 , other than 1 . This is a very interesting case which has been studied very little.

0.3. In an unpublished work, Bernstein (partly in collaboration with Zelevinskii) described a large commutative subalgebra of $H_{v_{0}}$ and the center of $H_{v_{0}}$ (in the "special case" above). We extend these results to the general case. A number of new difficulties arise, most of them caused by the simple coroots which are divisible by 2 . This extension is done in $\S 3$, based on the preparation of $\S \S 1$ and 2.

In $\S 4$, we introduce a filtration of an affine Hecke algebra and show that the corresponding graded algebra is the one introduced in [5]. $\S \S 5-7$ are again preparatory. In $\S 8$, we show that the completion of an affine Hecke algebra with respect to a maximal ideal of the center is isomorphic to the ring of $n \times n$ matrices with entries in the completion of a (usually smaller) affine Hecke algebra. In $\S 9$, we define a natural homomorphism from an affine Hecke algebra to a suitable completion of its graded version, which becomes an isomorphism when the first algebra is completed. This homomorphism is of the same nature as the Chern character from $K$-theory to homology. In $\S 10$, we combine the results of $\S \S 8$ and 9 to get our main result (see 10.9), comparing the representation theory of an affine Hecke algebra with that of a graded one.

0.4. Most of the work on this paper was done during the Fall of 1988 when I enjoyed the hospitality and support of the Institute for Advanced Study, Princeton. I acknowledge partial support of N.S.F. grant DMS-8610730 (at IAS) and DMS-8702842.

\section{CONTENTS}

1. Root systems and affine Weyl groups

2. The braid group

3. The Hecke algebra

4. The graded Hecke algebra

5. The elements $\tau_{w}$ and $\bar{\tau}_{w}$

6. Some braid group relations

7. Completions

8. First reduction theorem

9. Second reduction theorem

10. On simple $H$-modules

References

\section{ROOT SYSTEMS AND AFFINE WEYL GROUPS}

1.1. A root system $(X, Y, R, \check{R}, \Pi)$ consists of

(a) two free abelian groups of finite rank $X, Y$ with a given perfect pairing $\langle\rangle:, X \times Y \rightarrow \mathbf{Z}$, 
(b) two finite subsets $R \subset X, \check{R} \subset Y$ with a given bijection $R \leftrightarrow \check{R}$ denoted $\alpha \leftrightarrow \check{\alpha}$, and

(c) a subset $\Pi \subset R$.

These data are subject to requirements (d)-(f) below.

(d) $\langle\alpha, \check{\alpha}\rangle=2$ for all $\alpha \in R$.

(e) For any $\alpha \in R$, the reflection $s_{\alpha}: X \rightarrow X, x \mapsto x-\langle x, \check{\alpha}\rangle \alpha$ (resp. $s_{\alpha}: Y \rightarrow Y, y \rightarrow y-\langle\alpha, y\rangle \check{\alpha}$ ) leaves stable $R$ (resp. $\check{R}$ ).

(f) Any $\alpha \in R$ can be written uniquely as $\alpha=\sum_{\beta \in \Pi} n_{\alpha, \beta} \cdot \beta$, where $n_{\alpha, \beta} \in \mathbf{Z}$ are all $\geq 0$ or all $\leq 0$. (Accordingly, we say that $\alpha \in R^{+}$or $\alpha \in R^{-}$.)

Throughout this paper we will fix a root system $(X, Y, R, \check{R}, \Pi)$.

The subgroup of $\mathrm{GL}(X)$ generated by all $s_{\alpha}(\alpha \in R)$ may be identified with the subgroup of $\operatorname{GL}(Y)$ generated by all $s_{\alpha}(\alpha \in R)$ by $w \rightarrow{ }^{t} w^{-1}$. This is the Weyl group $W_{0}$ of the root system. It is a finite Coxeter group on generators $\left\{s_{\alpha} \mid \alpha \in \Pi\right\}$.

1.2. Consider the partial order $\leq$ on $\check{R}$ defined by $\check{\alpha}_{1} \leq \check{\alpha}_{2} \Leftrightarrow \check{\alpha}_{2}-\check{\alpha}_{1}$ is a linear combination with integer $\geq 0$ coefficients of elements of $\{\check{\alpha} \mid \alpha \in \Pi\}$.

Let $\Pi_{m}$ be the set of all $\beta \in R$ such that $\check{\beta}$ is a minimal element for $\leq$.

1.3. We shall assume throughout the paper that $(X, Y, R, \check{R}, \Pi)$ is reduced, i.e., $\alpha \in R \Rightarrow 2 \alpha \notin R$.

1.4. Let $W$ be the semidirect product $W_{0} \cdot X$. Its elements are $w a^{x} \quad(w \in$ $W, x \in X)$ with multiplication given by $w a^{x} \cdot w^{\prime} a^{x^{\prime}}=w w^{\prime} a^{w^{\prime-1}(x)+x^{\prime}} ; a$ is a fixed symbol. $W$ acts on $Y \times \mathbf{Z}$ by $w a^{x}:(y, k) \rightarrow(w(y), k-\langle x, y\rangle)$. Let $\widetilde{R}=\widetilde{R}^{+} \cup \widetilde{R}^{-} \subset Y \times \mathbf{Z}$ be defined by

$$
\begin{aligned}
& \widetilde{R}^{+}=\{(\check{\alpha}, k) \mid \alpha \in R, k>0\} \cup\left\{(\check{\alpha}, 0) \mid \alpha \in R^{+}\right\}, \\
& \widetilde{R}^{-}=\{(\check{\alpha}, k) \mid \alpha \in R, k<0\} \cup\left\{(\check{\alpha}, 0) \mid \alpha \in R^{-}\right\} .
\end{aligned}
$$

Then $\widetilde{R}$ is a $W$-stable subset of $Y \times \mathbf{Z}$.

Define $l: W \rightarrow \mathbf{N}$ by

(a)

$$
\begin{aligned}
l\left(w a^{x}\right) & =\#\left\{A \in \widetilde{R}^{+} \mid\left(w a^{x}\right)(A) \in \widetilde{R}^{-}\right\} \\
& =\sum_{\substack{\alpha \in R^{+} \\
w(\alpha) \in R^{-}}}|\langle x, \check{\alpha}\rangle+1|+\sum_{\substack{\alpha \in R^{+} \\
w(\alpha) \in R^{+}}}|\langle x, \check{\alpha}\rangle| .
\end{aligned}
$$

Let

$$
\begin{aligned}
\widetilde{\Pi} & =\{(\check{\alpha}, 0) \mid \alpha \in \Pi\} \cup\left\{(\check{\alpha}, 1) \mid \alpha \in \Pi_{m}\right\} \subset \widetilde{R}^{+}, \\
S & =\left\{s_{\alpha} \mid \alpha \in \Pi\right\} \cup\left\{s_{\alpha} a^{\alpha} \mid \alpha \in \Pi_{m}\right\} \subset W .
\end{aligned}
$$

We have an obvious bijection $\widetilde{\Pi} \leftrightarrow S\left(A \leftrightarrow s_{A}\right)$. An element of $S$ maps the corresponding element of $\widetilde{\Pi}$ to its negative and it maps the complement of this 
element in $\widetilde{R}^{+}$into itself. It follows that for $z \in W, A \in \widetilde{\Pi}$, we have (b)

$$
l\left(s_{A} z\right)= \begin{cases}l(z)+1, & \text { if } z^{-1}(A) \in \widetilde{R}^{+} \\ l(z)-1, & \text { if } z^{-1}(A) \in \widetilde{R}^{-}\end{cases}
$$

It is clear that

(c) $l(w)=l\left(w^{-1}\right) \quad(w \in W)$.

We deduce that for $x \in X, \alpha \in \Pi$, we have

(d1) $\langle x, \check{\alpha}\rangle>0 \Rightarrow l\left(s_{\alpha} a^{x}\right)=l\left(a^{x}\right)+1, l\left(a^{x} s_{\alpha}\right)=l\left(a^{x}\right)-1$.

(d2) $\langle x, \check{\alpha}\rangle<0 \Rightarrow l\left(s_{\alpha} a^{x}\right)=l\left(a^{x}\right)-1, l\left(a^{x} s_{\alpha}\right)=l\left(a^{x}\right)+1$.

(d3) $\langle x, \check{\alpha}\rangle=0 \Rightarrow l\left(s_{\alpha} a^{x}\right)=l(x)+1, l\left(a^{x} s_{\alpha}\right)=l(x)+1$.

(e) Let

$$
\begin{aligned}
X_{\mathrm{dom}} & =\{x \in X \mid\langle x, \check{\alpha}\rangle \geq 0, \forall \alpha \in \Pi\} \\
& =\left\{x \in X \mid l\left(s_{\alpha} a^{x}\right)=l\left(\alpha^{x}\right)+1, \forall \alpha \in \Pi\right\} .
\end{aligned}
$$

We have, using (a) and (e),

(f) $w \in W_{0}, x \in X_{\mathrm{dom}} \Rightarrow l\left(a^{x}\right)=\sum_{\alpha \in R^{+}}\langle x, \check{\alpha}\rangle, l\left(w a^{x}\right)=l(w)+l\left(a^{x}\right)$.

It follows that

(g) $x, x^{\prime} \in X_{\mathrm{dom}} \Rightarrow l\left(a^{x} \cdot a^{x^{\prime}}\right)=l\left(a^{x}\right)+l\left(a^{x^{\prime}}\right)$.

1.5. Let $Q$ be the subgroup of $X$ generated by $R$. The subgroup $W_{0} Q$ of $W$ is a Coxeter group with $S$ as the set of simple reflections, the length function being the restriction of $l$. This subgroup is normal in $W$ and admits a complement $\Omega=\{w \in W \mid w(\widetilde{\Pi})=\widetilde{\Pi}\}=\{w \in W \mid l(w)=0\}$. It is known that $\Omega$ is an abelian group, isomorphic to $X / Q$.

1.6. The notion of direct sum of root systems is defined in an obvious way. We say that $(X, Y, R, \check{R}, \Pi)$ is primitive if either (a) or (b) below holds.

(a) $\check{\alpha} \notin 2 Y$ for all $\alpha \in R$.

(b) There is a unique $\alpha \in \Pi$ such that $\check{\alpha} \in 2 Y$; moreover, $\left\{w(\alpha) \mid w \in W_{0}\right\}$ generate $X$.

Lemma 1.7. In general, $(X, Y, R, \check{R}, \Pi)$ is (uniquely) a direct sum of primitive root systems.

Proof. We may assume that we can find $\alpha \in \Pi$ such that $\check{\alpha} \in 2 Y$. Let $X^{\prime}$ be the subgroup of $X$ generated by $\left\{w(\alpha) \mid w \in W_{0}\right\}, Y^{\prime}$ the subgroup of $Y$ generated by $\left\{\frac{1}{2} w(\check{\alpha}) \mid w \in W_{0}\right\}$,

$$
\begin{aligned}
& X^{\prime \prime}=\left\{x \in X \mid\left\langle x, y^{\prime}\right\rangle=0, \forall y^{\prime} \in Y^{\prime}\right\}, \\
& Y^{\prime \prime}=\left\{y \in Y \mid\left\langle x^{\prime}, y\right\rangle=0, \forall x^{\prime} \in X^{\prime}\right\} .
\end{aligned}
$$

It is easy to see that $\langle$,$\rangle defines by restriction a perfect pairing X^{\prime} \times Y^{\prime} \rightarrow \mathbf{Z}$. Hence, we have $X=X^{\prime} \oplus X^{\prime \prime}, Y=Y^{\prime} \oplus Y^{\prime \prime}$, and $\langle$,$\rangle also defines a perfect$ pairing $X^{\prime \prime} \times Y^{\prime \prime} \rightarrow \mathbf{Z}$. Let $R^{\prime}=R \cap X^{\prime}, \check{R}^{\prime}=\check{R} \cap Y^{\prime}, \Pi^{\prime}=\Pi \cap X^{\prime}$, $R^{\prime \prime}=R \cap X^{\prime \prime}, \check{R}^{\prime \prime}=R \cap Y^{\prime \prime}, \Pi^{\prime \prime}=\Pi \cap X^{\prime \prime}$. It is clear that $(X, Y, R, \check{R}, \Pi)$ is a 
direct sum of the root systems $\left(X^{\prime}, Y^{\prime}, R^{\prime}, \check{R}^{\prime}, \Pi^{\prime}\right)$ and $\left(X^{\prime \prime}, Y^{\prime \prime}, R^{\prime \prime}, \check{R}^{\prime \prime}, \Pi^{\prime \prime}\right)$, the first of which is primitive. We can repeat this procedure if necessary and the lemma follows.

\section{THE BRAID GROUP}

2.1. Let $B$ be the group with generators $T_{w}(w \in W)$ and relations

(a) $T_{w} T_{w^{\prime}}=T_{w w^{\prime}}$ whenever $l(w)+l\left(w^{\prime}\right)=l\left(w w^{\prime}\right)$.

We say that $B$ is the braid group of $W$.

Lemma 2.2. Let $x \in X, \alpha \in \Pi$ be such that $\langle x, \check{\alpha}\rangle=0$. Let $s=s_{\alpha}$. Then $T_{a^{x}} T_{s}=T_{s} T_{a^{x}}$ in $B$.

Proof. Using 2.1(a) and 1.4(d3), we have $T_{a^{x}} T_{s}=T_{a^{x} s}=T_{s a^{x}}=T_{s} T_{a^{x}}$.

Lemma 2.3. Let $x \in X_{\text {dom }}, \alpha \in \Pi$ be such that $\langle x, \alpha\rangle=p>1$. Let $s=s_{\alpha}$, $w=s a^{x} s a^{x}$. Then

(a) $l\left(a^{x} s\right)=l\left(a^{x}\right)-1$.

(b) $l\left(a^{x} s a^{x}\right)=2 l\left(a^{x}\right)-2 p+1$.

(c) $l(w)=2 l\left(a^{x}\right)-2 p, w=a^{2 x-p \alpha}$, and $2 x-p \alpha \in X_{\mathrm{dom}}$.

(d) If $p=1$, then $T_{s}^{-1} T_{a^{x}} T_{s}^{-1} T_{a^{x}}=T_{w}$ in $B$.

Proof. Let $l(x)=\lambda$. Now (a) follows from 1.4(d1). We have $s a^{x} s a^{x}=$ $a^{s(x)+x}=a^{2 x-p \alpha},\langle 2 x-p \alpha, \breve{\beta}\rangle=2\langle x, \breve{\beta}\rangle-p\langle\alpha, \breve{\beta}\rangle \quad(\beta \in \Pi)$. If $\beta \neq \alpha$, then $\langle\alpha, \check{\beta}\rangle \leq 0$ so $\langle 2 x-p \alpha, \check{\beta}\rangle \geq 0$. If $\beta=\alpha$, then $\langle 2 x-p \alpha, \check{\beta}\rangle=2 p-2 p=0$. Hence, $2 x-\alpha \in X_{\text {dom }}$. By 1.4(f) we have

$$
\begin{aligned}
l\left(s a^{x} s a^{x}\right) & =\sum_{\beta \in R^{+}}\langle s(x)+x, \check{\beta}\rangle \\
& =\sum_{\beta \in R^{+}}\langle s(x), \check{\beta}\rangle+\sum_{\beta \in R^{+}}\langle x, \check{\beta}\rangle \\
& =\sum_{\beta \in R^{+}}\langle x, s(\check{\beta})\rangle+\sum_{\beta \in R^{+}}\langle x, \check{\beta}\rangle \\
& =2 \sum_{\beta \in R^{+}}\langle x, \check{\beta}\rangle-2\langle x, \check{\alpha}\rangle \\
& =2 l\left(a^{x}\right)-2 p \\
& =2 \lambda-2 p,
\end{aligned}
$$

hence $(\mathrm{c})$.

Now (b) follows from (c) using 1.4(d1).

From (a)-(c) we have (for $p=1$ )

$$
T_{s} T_{w}=T_{s w}=T_{a^{x} s a^{x}}, \quad T_{a^{x} s} T_{s}=T_{a^{x}}, \quad T_{a^{x} s} T_{a^{x}}=T_{a^{x} s a^{x}},
$$

hence (d).

2.4. We shall regard $S$ as the set of vertices of the Coxeter graph of $\left(W_{0} Q, S\right)$ in the usual way. For $\alpha \in \Pi$, let $S(\alpha) \subset S$ be the connected component of 
$s=s_{\alpha}$ in this graph. Assume that $\check{\alpha} \in 2 Y$. Then $S(\alpha)$ is a Coxeter graph of affine type $\widetilde{C}_{r}(r \geq 1)$. Hence, it has a unique nontrivial automorphism $\sim$ Let $\tilde{s}=\tilde{s}_{\alpha} \in S(\alpha)$. We can now state

Lemma 2.5. In the setup of 2.4, there is a unique element $x \in Q$ such that $\langle x, \check{\alpha}\rangle=2,\langle x, \check{\beta}\rangle=0$ for all $\beta \in \Pi-\{\alpha\}$. Moreover, there exist elements $w^{\prime}, w^{\prime \prime} \in W_{0} Q \subset W$ such that

$$
T_{a^{x} s a^{x}}=T_{w^{\prime} w^{\prime \prime}}, \quad T_{a^{2 x-\alpha}}=T_{w^{\prime}} T_{\tilde{s}} T_{w^{\prime \prime}}, \quad T_{a^{x} s} T_{a^{x}}=T_{w^{\prime}} T_{\tilde{s}}^{2} T_{w^{\prime \prime}} \quad \text { (in } B \text { ). }
$$

Proof. We may assume that $X=Q$ and $S=S(\alpha)$ (see 2.4). Let $Y_{1}$ be the subgroup (of index 2) of $Y$ generated by $\check{R}$. Let $X_{1}=\operatorname{Hom}\left(Y_{1}, Z\right)$. Then $X$ is naturally a subgroup (of index 2$)$ of $X_{1}$. Clearly, $\left(X_{1}, Y_{1}, R, \check{R}, \Pi\right)$ is a root system. We denote $W_{1}=W_{0} \cdot X_{1}$ with $l: W_{1} \rightarrow \mathbf{N}$ as in $1.4($ a) extending $l: W \rightarrow \mathbf{N}$ and let $B_{1}$ be the corresponding braid group. Then $W \subset W_{1}$, $B \subset B_{1}$ in a natural way; moreover, if $\Omega$ denotes the set of elements of length 0 of $W_{1}$, then $\Omega=\{1, \omega\}$ is a group of order 2 which is a complement of $W$ in $W_{1}$ and $\left\{1, T_{\omega}\right\}$ is a complement of $B$ in $B_{1}$. Now conjugation by $\omega$ is the nontrivial automorphism of $S$ (as a graph). Hence, $\omega s \omega^{-1}=\tilde{s}$ (see 2.4).

Let $x_{1} \in X_{1}$ be defined by $\left\langle x_{1}, \check{\alpha}\right\rangle=1,\left\langle x_{1}, \check{\beta}\right\rangle=0$ for $\beta \in \Pi-\{\alpha\}$. We have $x_{1} \notin X$. Indeed, if we had $x_{1} \in X$, then from $\check{\alpha} \in 2 Y$ it would follow that $\left\langle x_{1}, \check{\alpha}\right\rangle \in 2 \mathrm{Z}$, contradicting $\left\langle x_{1}, \check{\alpha}\right\rangle=1$.

Set $x=2 x_{1}$. Then $x \in X$, since $X$ has index 2 in $X_{1}$. Moreover, $\langle x, \check{\alpha}\rangle=$ $2,\langle x, \widehat{\beta}\rangle=0$ for $\beta \in \Pi-\{\alpha\}$.

Let $\omega^{\prime}-a^{x_{1}} s \omega \in W_{1}, w^{\prime \prime} \in \omega a^{3 x_{1}-\alpha} \in W_{1}$.

Since $x_{1} \in X_{1}-X$, we have $a^{x_{1}} \in \omega W, a^{3 x_{1}} \in \omega W$. Hence, $w^{\prime}, w^{\prime \prime} \in W$. We set $l_{1}=l\left(x_{1}\right)$. Applying Lemma 2.3 to $x_{1}$ instead of $x$, we see that

(a) $l\left(w^{\prime}\right)=l\left(a^{x_{1}} s\right)=l_{1}-1$.

(b) $l\left(w^{\prime \prime}\right)=l\left(a^{x_{1}} \cdot a^{2 x_{1}-\alpha}\right)=l\left(a^{x_{1}}\right)+l\left(a^{2 x_{1}-\alpha}\right)($ see $1.4(\mathrm{~g}))$

$$
=l_{1}+2 l_{1}-2=3 l_{1}-2 \text {. }
$$

(c) $l\left(a^{x}\right)=l\left(a^{x_{1}} \cdot a^{x_{1}}\right)=2 l\left(a^{x_{1}}\right)=2 l_{1}($ see $1.4(\mathrm{~g}))$.

(d) $l\left(a^{x_{1}} s\right)=l\left(a^{x}\right)-1=2 l_{1}-1($ see $1.4(\mathrm{~d} 1))$.

(e) $l\left(a^{x_{1}} s\right)=l\left(a^{x_{1}}\right)-1=l_{1}-1($ see $1.4(\mathrm{~d} 1))$.

(f) $l\left(a^{2 x-\alpha}\right)=l\left(a^{2 x_{1}} \cdot a^{2 x_{1}-\alpha}\right)=l\left(a^{2 x_{1}}\right)+l\left(a^{2 x_{1}-\alpha}\right)=2 l_{1}+2 l_{1}-2=4 l_{1}-2$.

Applying Lemma 2.3(b) to $x$, we see that

(g) $l\left(a^{x} s a^{x}\right)=4 l_{1}-1-3$.

Next we note

$$
\begin{aligned}
w^{\prime} w^{\prime \prime} & =a^{x_{1}} s a^{3 x_{1}-\alpha}=a^{x_{1}} a^{s\left(x_{1}\right)} s a^{2 x_{1}-\alpha} \\
& =a^{2 x_{1}-\alpha} s a^{2 x_{1}-\alpha}=a^{2 x_{1}} s a^{\alpha} a^{2 x_{1}-\alpha} \\
& =a^{2 x_{1}} s a^{2 x_{1}}=a^{x} s a^{x} .
\end{aligned}
$$

By (a), (b), and (g) we have $l\left(w^{\prime}\right)+l\left(w^{\prime \prime}\right)=l\left(a^{x} s a^{x}\right)=4 l_{1}-3$. Hence, $T_{w^{\prime}} T_{w^{\prime \prime}}=T_{a^{x} s a^{x}}$. 
We have

$$
w^{\prime} \tilde{s} w^{\prime \prime}=a^{x_{1}} s \omega \tilde{s} \omega a^{3 x_{1}-\alpha}=a^{x_{1}} \cdot a^{3 x_{1}-\alpha}=a^{2 x-\alpha} .
$$

By (a), (b), and (f) we have $l\left(w^{\prime}\right)+l(\tilde{s})+l\left(w^{\prime \prime}\right)=l\left(a^{2 x-\alpha}\right)=4 l_{1}-2$.

We have

$$
\begin{aligned}
T_{a^{x_{s}}} T_{a^{x}} & =T_{a^{x_{1}}} T_{a^{x_{1}} s} T_{a^{x_{1}}} T_{a^{x_{1}}} \quad(\text { see }(\mathrm{d}),(\mathrm{e})) \\
& =T_{a^{x_{1}}} T_{a^{x_{1}}} T_{s}^{-1} T_{a^{x_{1}}} T_{a^{x_{1}}} \quad(\text { see } 1.4(\mathrm{~d} 1)) \\
& =T_{a^{x_{1}}} T_{s} T_{s a^{x_{1}} s a^{x_{1}}} \quad(\text { see Lemma } 2.3(\mathrm{~d})) \\
& =T_{a^{x_{1}}} T_{s} T_{s a^{x_{1}} s a^{x_{1}} a^{x_{1}}} \quad(\text { see } 1.4(\mathrm{~g})) \\
& =T_{a^{x_{1}} s} T_{s} T_{s} T_{a^{3 x_{1}-\alpha}} \quad(\text { see } 1.4(\mathrm{~d} 1)) \\
& =T_{w^{\prime}} T_{\omega} T_{s} T_{s} T_{\omega} T_{w^{\prime \prime}} \\
& =T_{w^{\prime}} T_{\tilde{s}}^{2} T_{w^{\prime \prime}} .
\end{aligned}
$$

The lemma is proved.

2.6. We define, for any $x \in X$, an element $\bar{T}_{x} \in B$ as follows. We write $x=x_{1}-x_{2}$ with $x_{1}, x_{2} \in X_{\text {dom }}$, and we set $\bar{T}_{x}=T_{a^{x_{1}}} T_{a^{x_{2}}}^{-1}$. This is independent of the choice of $x_{1}, x_{2}$ since $T_{a^{x^{\prime}}} T_{a^{x^{\prime \prime}}}=T_{a^{x^{\prime \prime}}} T_{a^{x^{\prime}}}=T_{a^{x^{\prime}+x^{\prime \prime}}}$ for $x^{\prime}, x^{\prime \prime} \in X_{\mathrm{dom}}$ (see 1.4(g)).

Lemma 2.7. (a) If $x \in X_{\text {dom }}$, then $\bar{T}_{x}=T_{a^{x}}$.

(b) We have $\bar{T}_{x}, \bar{T}_{x^{\prime \prime}}=\bar{T}_{x^{\prime}+x^{\prime \prime}}$ for $x^{\prime}, x^{\prime \prime} \in X$.

(c) If $x \in X, \alpha \in \Pi$ satisfy $\langle x, \check{\alpha}\rangle=0$ and $s_{\alpha}=s$, then $T_{s} \bar{T}_{x}=\bar{T}_{x} T_{s}$.

(d) If $x \in X, \alpha \in \Pi$ satisfy $\langle x, \check{\alpha}\rangle=1$ and $s_{\alpha}=s$, then $\bar{T}_{x}=T_{s} \bar{T}_{s(x)} T_{s}$.

(e) If $x \in X, \alpha \in \Pi$ satisfy $\langle x, \check{\alpha}\rangle=2, \check{\alpha} \in 2 Y$, and $s_{\alpha}=s$, then there exist elements $\gamma, \gamma^{\prime} \in B$ such that $T_{s} \bar{T}_{s(x)}=\gamma \gamma^{\prime}, \bar{T}_{x-\alpha}=\gamma T_{\tilde{s}} \gamma^{\prime}$, $\bar{T}_{x} T_{s}^{-1}=\gamma T_{\tilde{s}}^{2} \gamma^{\prime} ;(\tilde{s}$ as in 2.4).

Proof. (a) and (b) follow immediately from the definition. In (c), we can write $x=x_{1}-x_{2}$, where $x_{1}, x_{2} \in X_{\text {dom }}$ satisfy $\left\langle x_{1}, \check{\alpha}\right\rangle=\left\langle x_{2}, \check{\alpha}\right\rangle=0$, and it remains to observe that $T_{a^{x_{1}}}, T_{a^{x_{2}}}$ commute with $T_{s}$ by Lemma 2.2.

In (d), we can write $x=x_{1}-x_{2}$, where $x_{1}, x_{2} \in X_{\text {dom }}$ satisfy $\left\langle x_{1}, \check{\alpha}\right\rangle=1$, $\left\langle x_{2}, \check{\alpha}\right\rangle=0$. We have

$$
\begin{aligned}
T_{s}^{-1} \bar{T}_{x} T_{s}^{-1} \bar{T}_{x} & =T_{s}^{-1} T_{a^{x_{1}}} T_{a^{x_{2}}}^{-1} T_{s}^{-1} T_{a^{x_{1}}} T_{a^{x_{2}}}^{-1} \\
& \left.=T_{s}^{-1} T_{a^{x_{1}}} T_{s}^{-1} T_{a^{x_{1}}} T_{a^{x_{2}}}^{-2} \quad \text { (using Lemma 2.2 for } x_{2}\right) \\
& =T_{s a^{x_{1}} s a^{x_{1}}} T_{a^{2 x_{2}}}^{-1} \quad \text { (using Lemma 2.3(d)) } \\
& =T_{a^{2 x_{1}-\alpha}} T_{a^{2 x_{2}}}^{-1}=\bar{T}_{2 x-\alpha} \quad \text { (see Lemma 2.3(c)) } \\
& =\bar{T}_{2 x_{1}-\alpha-2 x_{2}}=\bar{T}_{s(x)} \cdot \bar{T}_{x} \quad(\text { see }(\mathrm{b})) .
\end{aligned}
$$

We now multiply on the right by $\bar{T}_{x}^{-1}$ and (d) follows. 
We fix $\alpha \in \Pi$ with $\check{\alpha} \in 2 Y$.

Assume that (e) holds for some $x \in X$ with $\langle x, \check{\alpha}\rangle=2$.

Now let $x^{\prime} \in X$ be such that $\left\langle x^{\prime}, \check{\alpha}\right\rangle=0$. Then (e) holds for $x+x^{\prime}$ instead of $x$, with $\bar{T}_{x^{\prime}} \gamma, \gamma^{\prime}$ instead of $\gamma, \gamma^{\prime}$ (we use (b) and (c)). Thus, it is enough to prove (e) for a single $x$, namely, that given by Lemma 2.5 . In this case, we set $\gamma=T_{w^{\prime}}, \gamma^{\prime}=T_{w^{\prime \prime}} T_{a^{x}}^{-1}$, where $w^{\prime}, w^{\prime \prime}$ are as in Lemma 2.5. Using Lemma 2.5, we are reduced to verifying the following identities:

(f) $T_{a^{x} s a^{x}} \cdot T_{a^{x}}^{-1}=T_{s} \bar{T}_{s(x)}$.

(g) $T_{a^{2 x-\alpha}} T_{a^{x}}^{-1}=\bar{T}_{x-\alpha}$.

(h) $T_{a^{x} s}=\bar{T}_{x} T_{s}^{-1}$.

Now $T_{s}^{-1} T_{a^{x} s a^{x}}=T_{s a^{x} s a^{x}}$ (see Lemma 2.3(b) and (c)) and $s a^{x} s a^{x}=a^{s(x)+x}$. Hence, (f) is equivalent to $T_{a^{s(x)+x}} \cdot T_{a^{x}}^{-1}=\bar{T}_{s(x)}$ which follows from the definition of $\bar{T}_{s(x)}$ and the fact that $s(x)+x=2 x-2 \alpha \in X_{\text {dom }}$ (see Lemma 2.3(c)). We also have that $x-\alpha \in X_{\text {dom }}$. Hence, $\bar{T}_{x-\alpha}=T_{a^{x-\alpha}}$ and (g) is equivalent to $T_{a^{x}} \cdot T_{a^{x-\alpha}}=T_{a^{2 x-\alpha}}$, which follows from $1.4(\mathrm{~g})$. Finally, (h) is equivalent to $T_{a^{x} s} T_{s}=T_{a^{x}}$ which follows from 1.4(d1). The lemma is proved.

Lemma 2.8. Let $B^{\prime}$ be the subgroup of $B$ generated by $T_{x}$ for all $x \in X_{\mathrm{dom}}$ and by $T_{s_{\alpha}}$ for all $\alpha \in \Pi$. Then $B^{\prime}=B$.

Proof. Let $\alpha \in \Pi_{m}$, let $A=(\check{\alpha}, 1) \in \widetilde{\Pi}, s_{A}=s_{\alpha} a^{\alpha} \in S$ (see 1.4). Now $-\alpha \in$ $X_{\text {dom }}$ (since $\left.\alpha \in \Pi_{m}\right)$. We have $a^{\alpha}(A)=a^{\alpha}(\check{\alpha}, 1)=(\check{\alpha},-1) \in \widetilde{R}^{-}$. Hence, by 1.4(b), we have $l\left(s_{A} a^{-\alpha}\right)=l\left(a^{-\alpha}\right)-1$. This implies $T_{a^{-\alpha}}=T_{s_{A}} T_{s_{A} a^{-\alpha}}=T_{s_{A}} T_{s_{\alpha}}$. Hence, $T_{s_{A}}=T_{a^{-\alpha}} T_{s_{\alpha}}^{-1} \in B^{\prime}$. Thus, $T_{s} \in B^{\prime}$ for all $s \in S$. Hence, $T_{w} \in B^{\prime}$ for all $w \in W_{0} Q$, since such $w$ are of the form $s_{1} s_{2} \cdots s_{p} \quad\left(s_{i} \in S, p=l(w)\right)$. Now let $w \in \Omega$. We can find $x \in X_{\text {dom }}$ such that $x \cdot \omega \in W_{0} Q$ and $T_{x \omega} \in B^{\prime}$. We have $T_{x} T_{\omega}=T_{x \omega}$. Hence, $T_{\omega}=T_{x}^{-1} \cdot T_{x \omega} \in B^{\prime}$. Since $B$ is generated by the elements $T_{\omega}(\omega \in \Omega)$ and $T_{w}\left(w \in W_{0} Q\right)$, we have $B^{\prime}=B$.

\section{The Hecke Algebra}

3.1. Consider the following three kinds of data:

(a) a function $L: W \rightarrow \mathbf{N}$ such that $L\left(w w^{\prime}\right)=L(w)+L\left(w^{\prime}\right)$ whenever $w, w^{\prime} \in W$ satisfy $l\left(w w^{\prime}\right)=l(w)+l\left(w^{\prime}\right)$,

(b) a function $L_{1}: S \rightarrow \mathbf{N}$ such that $L_{1}(s)=L_{1}\left(s^{\prime}\right)$ whenever $s, s^{\prime} \in S$ are conjugate in $W$,

(c) a function $\lambda: \Pi \rightarrow \mathbf{N}$ such that $\lambda(\alpha)=\lambda\left(\alpha^{\prime}\right)$ whenever $\alpha, \alpha^{\prime} \in \Pi$ satisfy $\left\langle\alpha^{\prime}, \check{\alpha}\right\rangle=\left\langle\alpha, \check{\alpha}^{\prime}\right\rangle=-1$, together with a function $\lambda^{*}:\{\alpha \in \Pi \mid \check{\alpha} \in$ $2 Y\} \rightarrow \mathbf{N}$.

We have a natural bijection between the set of functions as in (a) and the set of functions as in (b) and also a natural bijection between the set of functions as in (b) and the set of pairs of functions as in (c). The first bijection is defined 
by $L \rightarrow L_{1}=\left.L\right|_{S}$, the second by $L_{1} \rightarrow\left(\lambda, \lambda^{*}\right)$, where $\lambda(\alpha)=L_{1}\left(s_{\alpha}\right) \quad(\alpha \in \Pi)$, $\lambda^{*}(\alpha)=L_{1}\left(\tilde{s}_{\alpha}\right) \quad\left(\alpha \in \Pi, \check{\alpha} \in 2 Y, \tilde{s}_{\alpha}\right.$ as in 2.4). To see that it is a bijection, we use Lemma 1.7 .

Hence, a datum of type (a) is equivalent to a datum of type (b) or one of type (c). Such a datum is called a parameter set for the root system.

We shall assume that a parameter set for our root system has been fixed. Hence, $L, L_{1}, \lambda$, and $\lambda^{*}$ are defined.

We denote by $\widetilde{L}: B \rightarrow \mathbf{Z}$ the unique homomorphism such that $\widetilde{L}\left(T_{w}\right)=$ $L(w)$ for all $w \in W$.

Many results in this section are due to Bernstein and Zelevinski, or Bernstein, in the "special case"mentioned in 0.1 (see $[4,4.3,4.4]$ ).

3.2. Let $v$ be an indeterminate and let $\mathscr{A}=\mathbf{C}\left[v, v^{-1}\right]$. The Hecke algebra $H$ (over $\mathscr{A}$ ) is defined to be the quotient of the group algebra (over $\mathscr{A}$ ) of the braid group $B$ by the two-sided ideal generated by the elements

$$
\left(T_{s}+1\right)\left(T_{s}-v^{2 L(s)}\right), \quad s \in S .
$$

The image of $T_{w} \in B$ (resp. $\bar{T}_{x} \in B$, see 2.6) in $H$ is denoted again $T_{w}$ (resp. $\bar{T}_{x}$ ).

It is well known that

(a) the elements $T_{w} \in H \quad(w \in W)$ form a basis of $H$ as an $\mathscr{A}$-module. We have the following identity in $H$ :

(b) $\left(T_{s}+1\right)\left(T_{s}-v^{2 L(s)}\right)=0 \quad(s \in S)$.

3.3. For any $x \in X$, we define

(a) $\theta_{x}=v^{-\widetilde{L}\left(\bar{T}_{x}\right)} \bar{T}_{x} \in H$.

From Lemma 2.7(b) we see that

(b) $\theta_{x} \theta_{x^{\prime}}=\theta_{x+x^{\prime}}$ for all $x, x^{\prime} \in X, \theta_{0}=1$.

Lemma 3.4. (a) The elements $T_{w} \theta_{x} \in H \quad\left(w \in W_{0}, x \in X\right)$ are linearly independent over $\mathscr{A}$.

(b) The elements $\theta_{x} T_{w} \in H \quad\left(w \in W_{0}, x \in X\right)$ are linearly independent over $\mathscr{A}$.

Proof. Assume that we have a relation $\sum_{i=1}^{n} f_{i} T_{w_{i}} \theta_{x_{i}}=0$, where $\left(w_{1}, x_{1}\right), \ldots$, $\left(w_{n}, x_{n}\right)(n \geq 1)$ are distinct elements of $W_{0} \times X$ and $f_{1}, \ldots, f_{n} \in \mathscr{A}-0$. We can find $x \in X_{\text {dom }}$ such that $x+x_{i} \in X_{\text {dom }}$ for $i=1, \ldots, n$. Multiplying our relation on the right by $\theta_{x}$, we obtain $\sum_{i=1}^{n} f_{i} T_{w_{i}} \theta_{x_{i}+x}=0$. Hence,

$$
\sum_{i=1}^{n} f_{i} v^{-L\left(a^{x+x_{i}}\right)} T_{w_{i}} T_{a^{x+x_{i}}}=0
$$

Using 1.4(f), we deduce $\sum_{i=1}^{n} f_{i} v^{-L\left(\alpha^{x+x_{i}}\right)} T_{w_{i} a^{x+x_{i}}}=0$, contradicting 3.2(a). This proves (a). The proof of $(b)$ is similar and will be omitted. 
3.5. Let $\mathscr{O}$ be the $\mathscr{A}$-submodule of $H$ generated by the elements $\theta_{x} \quad(x \in$ $X)$. This is a subalgebra of $H$. From Lemma 3.4, we see that

(a) $\mathscr{A}[X] \rightarrow \mathscr{O},\left(x \mapsto \theta_{x}\right)$ is an $\mathscr{A}$-algebra isomorphism. $(\mathscr{A}[X]$ is the group algebra of $X$ over $\mathscr{A}^{x}$.)

(b) Let $K$ be the quotient field of the algebra $\mathscr{O}$. If $x \in X$ and $\alpha \in \Pi$, the element $\left(\theta_{x}-\theta_{s_{\alpha}(x)}\right) /\left(1-\theta_{-\alpha}\right) \in K$ actually belongs to $\mathscr{O}$. Indeed, it is equal to $\theta_{x}\left(1-\theta_{-\alpha}^{n}\right) /\left(1-\theta_{-\alpha}\right)$, where $n=\langle x, \check{\alpha}\rangle$. Similarly, if $\alpha \in \Pi$ is such that $\check{\alpha} \in 2 Y$, then the element $\left(\theta_{x}-\theta_{s_{\alpha}(x)}\right) /\left(1-\theta_{-2 \alpha}\right) \in K$ actually belongs to $\mathscr{O}$. It is equal to $\theta_{x}\left(1-\theta_{-2 \alpha}^{n^{\prime}}\right) /\left(1-\theta_{-2 \alpha}\right)$, where $n^{\prime}=\frac{1}{2}\langle x, \check{\alpha}\rangle \in \mathbf{Z}$. We can now state the following result.

Proposition 3.6. Let $x \in X, \alpha \in \Pi, s=s_{\alpha}$. We have the following identity in $H$ :

$$
\theta_{x} T_{s}-T_{s} \theta_{s(x)}=\left\{\begin{array}{l}
\left(v^{2 \lambda(\alpha)}-1\right) \frac{\theta_{x}-\theta_{s(x)}}{1-\theta_{-\alpha}}, \quad \text { if } \check{\alpha} \notin 2 Y, \\
\left(\left(v^{2 \lambda(\alpha)}-1\right)+\theta_{-\alpha}\left(v^{\lambda(\alpha)+\lambda^{*}(\alpha)}-v^{\lambda(\alpha)-\lambda^{*}(\alpha)}\right)\right) \frac{\theta_{x}-\theta_{s(x)},}{1-\theta_{-2 \alpha}}, \\
\text { if } \check{\alpha} \in 2 Y .
\end{array}\right.
$$

(Recall that $\lambda(\alpha)=L(s), \lambda^{*}(\alpha)=L(\tilde{s})$, where $\tilde{s}=\tilde{s}_{\alpha}$ is as in 2.4.)

Proof. Assume that $\alpha$ is fixed and that the identity above is known for two elements $x, x^{\prime}$ of $X$. Then we see immediately that it also holds for $x+x^{\prime}$ and for $-x$. Hence, it is enough to prove the identity for $x$ in a fixed set of generators of the abelian group $X$.

If $\check{\alpha} \notin 2 Y$, we can find $x_{1} \in X$ such that $\left\langle x_{1}, \check{\alpha}\right\rangle=1$, and $X$ is generated by $x_{1}$ and by the elements $x^{\prime} \in X$ such that $\left\langle x^{\prime}, \check{\alpha}\right\rangle=0$.

If $\check{\alpha} \in 2 Y$, we can find $x_{2} \in X$ such that $\left\langle x_{2}, \check{\alpha}\right\rangle=2$, and $X$ is generated by $x_{2}$ and by the elements $x^{\prime} \in X$ such that $\left\langle x^{\prime}, \check{\alpha}\right\rangle=0$.

If $\langle x, \check{\alpha}\rangle=0$, we have $s x=x$, and our identity reduces to $\theta_{x} T_{s}=T_{s} \theta_{x}$ which follows from Lemma 2.7 (c). Therefore, it remains to prove our identity for $x \in X$ such that

$$
\langle x, \hat{\alpha}\rangle= \begin{cases}1, & \text { if } \check{\alpha} \notin 2 Y, \\ 2, & \text { if } \check{\alpha} \in 2 Y .\end{cases}
$$

Assume first that $\check{\alpha} \notin 2 Y$ and $\langle x, \check{\alpha}\rangle=1$. From Lemma $2.7(\mathrm{~d})$, we have $\bar{T}_{x}=T_{s} \bar{T}_{s(x)} T_{s}$ in $B$. Applying $\widetilde{L}$ to this, we obtain $\widetilde{L}\left(\bar{T}_{x}\right)=\widetilde{L}\left(T_{s(x)}\right)+2 L(s)$. Hence, $v^{\widetilde{L}\left(\bar{T}_{x}\right)} \theta_{x}=T_{s} v^{\widetilde{L}\left(\bar{T}_{s(x)}\right)} \theta_{s(x)} T_{s}$ in $H$, so that $v^{2 L(s)} \theta_{x} T_{s}^{-1}=T_{s} \theta_{s(x)}$. We substitute $T_{s}^{-1}=v^{-2 L(s)} T_{s}+\left(v^{-2 L(s)}-1\right)\left(\right.$ see 3.2(b)), and we obtain $\theta_{x} T_{s}-$ $T_{s} \theta_{s(x)}=\left(v^{2 L(s)}-1\right) \theta_{x}$ which verifies the desired identity.

Assume next that $\check{\alpha} \in 2 Y$ and $\langle x, \check{\alpha}\rangle=2$. From Lemma 2.7(e), we have (a) $T_{s} \bar{T}_{s(x)}=\gamma \gamma^{\prime}, \bar{T}_{x-\alpha}=\gamma T_{\tilde{s}} \gamma^{\prime}, \bar{T}_{x} T_{s}^{-1}=\gamma T_{\tilde{s}}^{2} \gamma^{\prime}$ for some $\gamma, \gamma^{\prime} \in B$.

Applying $\widetilde{L}$, we obtain

(b) $\widetilde{L}\left(\bar{T}_{s(x)}\right)=\nu_{0}-L(s), \widetilde{L}\left(\bar{T}_{x-a}\right)=\nu_{0}+L(\tilde{s}), \widetilde{L}\left(\bar{T}_{x}\right)=\nu_{0}+L(s)+2 L(\tilde{s})$, where $\nu_{0}=\widetilde{L}(\gamma)+\widetilde{L}\left(\gamma^{\prime}\right)$. 
In the group algebra of $B$ over $\mathscr{A}$ we have

$$
\bar{T}_{x} T_{s}^{-1}-\left(v^{2 L(\tilde{s})}-1\right) \bar{T}_{x-\alpha}-v^{2 L(\tilde{s})} T_{s} \bar{T}_{s(x)}=\gamma\left(T_{\tilde{s}}^{2}-\left(v^{2 L(\tilde{s})}-1\right) T_{\tilde{s}}-v^{2 L(\tilde{s})}\right) \gamma^{\prime} .
$$

The last expression has image equal to zero in $H$ (see 3.2(b)). Hence, in $H$ we have $\bar{T}_{x} T_{s}^{-1}-\left(v^{2 L(\hat{s})}-1\right) \bar{T}_{x-\alpha}-v^{2 L(\tilde{s})} T_{s} \bar{T}_{s(x)}=0$, or equivalently

$$
v^{\widetilde{L}\left(\bar{T}_{x}\right)} \theta_{x} T_{s}^{-1}-\left(v^{2 L(\tilde{s})}-1\right) v^{\widetilde{L}\left(\bar{T}_{x-a)}\right.} \theta_{x-\alpha}-v^{2 L(\tilde{s})} v^{\widetilde{L}\left(\bar{T}_{s(x)}\right)} T_{s} \theta_{s(x)}=0 .
$$

Using (b), this becomes

$$
v^{L(s)+2 L(\tilde{s})} \theta_{x} T_{s}^{-1}-\left(v^{2 L(\tilde{s})}-1\right) v^{L(\tilde{s})} \theta_{x-\alpha}-v^{2 L(\tilde{s})-L(s)} T_{s} \theta_{s(x)}=0 .
$$

We again substitute $T_{s}^{-1}=v^{-2 L(s)} T_{s}+\left(v^{-2 L(s)}-1\right)$, and we obtain

$$
\theta_{x} T_{s}-T_{s} \theta_{s(x)}=\left(v^{L(s)+L(\tilde{s})}-v^{L(s)-L(\tilde{s})}\right) \theta_{x-\alpha}+\left(v^{2 L(s)}-1\right) \theta_{x}
$$

which verifies the desired identity. This completes the proof of the proposition.

Proposition 3.7. (a) The elements $T_{w} \theta_{x} \in H \quad\left(w \in W_{0}, x \in X\right)$ form an $\mathscr{A}$-basis for $H$.

(b) The elements $\theta_{x} T_{w} \in H \quad\left(w \in W_{0}, x \in X\right)$ form an $\mathscr{A}$-basis for $H$.

Proof. Let $H_{1}$ (resp. $H_{2}$ ) be the $\mathscr{A}$-submodule of $H$ generated by the elements in (a) (resp. in (b)). Using Proposition 3.6 and induction on $l(w)$, we see that $T_{w} \theta_{x} \in H_{2}, \theta_{x} T_{w} \in H_{1}$ for any $w \in W_{0}, x \in X$. Hence, $H_{1}=H_{2}$. Now $H_{1}$ is stable under left multiplication by elements $T_{w}$, while $H_{2}$ is stable under left multiplication by elements $\theta_{x}$. Hence, $H_{1}=H_{2}$ is stable under left multiplication by elements $T_{w}, \theta_{x}\left(w \in W_{0}, x \in X\right)$. But these elements generate $H$ as an $\mathscr{A}$-algebra, by Lemma 2.8, and $1 \in H_{1}=H_{2}$. It follows that $H_{1}=H_{2}=H$. It remains to use Lemma 3.4.

3.8. We define for any $\alpha \in \Pi$ an element $\mathscr{G}(\alpha) \in K$ (see 3.5(b)) by

$$
\mathscr{G}(\alpha)=\left\{\begin{array}{l}
\frac{\theta_{\alpha} v^{2 \lambda(\alpha)}-1}{\theta_{\alpha}-1}, \quad \text { if } \check{\alpha} \notin 2 Y, \\
\frac{\left(\theta_{\alpha} v^{\lambda(\alpha)+\lambda^{*}(\alpha)}-1\right)\left(\theta_{\alpha} v^{\lambda(\alpha)-\lambda^{*}(\alpha)}+1\right)}{\theta_{2 \alpha}-1}, \quad \text { if } \check{\alpha} \in 2 Y .
\end{array}\right.
$$

(This is reminiscent of the $c_{0}$-function in [6, p. 51].)

We can reformulate the identity in Proposition 3.6 as follows.

Proposition 3.9. In the setup of Proposition 3.6 we have

$$
\theta_{x}\left(T_{s}+1\right)-\left(T_{s}+1\right) \theta_{s(x)}=\left(\theta_{x}-\theta_{s(x)}\right) \mathscr{G}(\alpha) \text {. }
$$

(The right-hand side is in $\mathcal{O}$.)

Corollary 3.10. If $x \in X, \alpha \in \Pi$, then $\theta_{x}+\theta_{s_{\alpha}(x)}$ commutes with $T_{s_{\alpha}}$.

Proof. We write the identity in Proposition 3.9 for $x$ and for $s_{\alpha}(x)$ and add the results. We see that $\theta_{x}+\theta_{s_{\alpha}(x)}$ commutes with $T_{s_{\alpha}}+1$, hence the corollary.

The following result is due to Bernstein, in the "special case" (see 0.1). 
Proposition 3.11. Let $\mathscr{Z}$ be the center of $H$. Then $\mathscr{Z}$ is the free $\mathscr{A}$-submodule of $H$ with basis $\left(z_{M}=\sum_{x \in M} \theta_{x}\right)$, where $M$ runs over all $W_{0}$-orbits in $X$.

Proof. From Corollary 3.10, we see that $z_{M}$ commutes with $T_{s_{\alpha}}$ for any $\alpha \in \Pi$. It clearly commutes with $\theta_{x^{\prime}}$ for any $x^{\prime} \in X$. Hence, by $2.8, z_{M}$ is in $\mathscr{Z}$. The linear independence of the elements $z_{M}$ is clear from 3.5(a). The fact that they generate $\mathscr{Z}$ as an $\mathscr{A}$-module is proved by a specialization argument (reduction by $v \rightarrow 1$ to the case of the group algebra $\mathbf{C}[W]$ ) as in $[3,8.1]$.

3.12. We have a natural $\mathscr{A}$-linear action $w: f \rightarrow w(f)$ of $W_{0}$ on $\mathscr{O}$ such that $w\left(\theta_{x}\right)=\theta_{w(x)}$ for $x \in X$, and the previous proposition implies that $\mathscr{Z}=\mathscr{O}^{W_{0}}$ (the $W_{0}$-invariants). This action extends to an action $w: f \rightarrow w(f)$ of $W_{0}$ on $K$ (by field automorphism). Let $F$ be the quotient field of $\mathscr{Z}$. We have

$$
\begin{aligned}
& \mathscr{Z} \subset \mathscr{O} \\
& \cap \cap \\
& F \subset K
\end{aligned}
$$

We have a natural isomorphism

(a) $\mathscr{O} \otimes_{\mathscr{Z}} F \stackrel{\approx}{\rightarrow} K, \xi \otimes f \mapsto \xi f$.

(We must show only that, given any element $\xi \in \mathscr{O}-0$, we can find $\xi^{\prime} \in \mathscr{O}-0$ such that $\xi \xi^{\prime} \in \mathscr{Z}$. It is sufficient to take $\xi^{\prime}=\Pi_{w \in W_{0} ; w \neq 1} w(\xi)$.)

Let $H_{F}$ be the $F$-algebra $H \otimes_{\mathscr{Z}} F$. It contains $H$ as a $\mathscr{Z}$-subalgebra. We identify the subspace $\mathscr{O} \otimes_{\mathscr{X}} F$ of $H_{F}$ with $K$ using (a). Thus, we have $K \subset H_{F}$.

We have two decompositions

(b) $H=\bigoplus_{w \in W_{0}} T_{w} \mathscr{O}=\bigoplus_{w \in W_{0}} \mathscr{O} T_{w}$

(cf. Proposition 3.7). Tensoring with $F$ over $\mathscr{Z}$, we obtain two decompositions,

(c) $H_{F}=\bigoplus_{w \in W_{0}} T_{w} K=\bigoplus_{w \in W_{0}} K T_{w}$.

In the $F$-algebra $H_{F}$, we have

(d) $f\left(T_{s_{\alpha}}+1\right)-\left(T_{s_{\alpha}}+1\right) s_{\alpha}(f)=\left(f-s_{\alpha}(f)\right) \mathscr{G}(a)$

for any $f \in K, \alpha \in \Pi$. Indeed, by (a), we can write $f=f_{1} / z, f_{1} \in \mathscr{O}$, $z \in \mathscr{Z}-0$ and we are reduced to the case where $f \in \mathscr{O}$, in which case we may use Proposition 3.9.

3.13. For later reference, we define for any $\alpha \in R: \lambda(\alpha)=\lambda\left(\alpha^{\prime}\right), \mathscr{G}(\alpha)=$ $w\left(\mathscr{G}\left(\alpha^{\prime}\right)\right)$, where $\alpha^{\prime}$ is a root in $\Pi$ and $w \in W_{0}$ is such that $\alpha=w\left(\alpha^{\prime}\right)$. (It is easy to see that this is independent of the choice of $\alpha^{\prime}$ and $w$.) Similarly, for any $\alpha \in R$ such that $\check{\alpha} \in 2 Y$, we set $\lambda^{*}(\alpha)=\lambda^{*}\left(\alpha^{\prime}\right)$, where $\alpha^{\prime} \in \Pi$, $w \in W_{0}, \alpha=w\left(\alpha^{\prime}\right)$. Then the formula for $\mathscr{G}(\alpha)$ given in 3.8 remains true for any $\alpha \in R$.

3.14. Let $\mathscr{T}$ be the torus $Y \otimes \mathbf{C}^{*}$. If $x \in X$, we shall identify the basis element $\theta_{x}$ of $\mathscr{O}$ with the character $\theta_{x}: \mathscr{T} \rightarrow \mathbf{C}^{*}, \theta_{x}(y \otimes \zeta)=\zeta^{\langle x, y\rangle} \quad(y \in Y$, $\left.\zeta \in \mathbf{C}^{*}\right)$. 
Hence, we may identify the $\mathbf{C}$-algebra $\mathscr{O}$ with the coordinate ring of the torus $T \times \mathbf{C}^{*}$ : to the basis element $v^{i} \theta_{x}$ of $\mathscr{O}$ corresponds the character $T \times \mathbf{C}^{*} \rightarrow \mathbf{C}^{*}$, $\left(t, \zeta_{1}\right) \mapsto \zeta_{1}^{i} \theta_{x}(t)$. In particular, $v$ corresponds to $\mathrm{pr}_{2}: \mathscr{T} \times \mathbf{C}^{*} \rightarrow \mathbf{C}^{*}$. Then $K$ becomes the field of rational functions on $\mathscr{T} \times \mathbf{C}^{*}$. We may also identify $Y$ with the group of all algebraic homomorphisms $\mathbf{C}^{*} \rightarrow \mathscr{T}$; to $y \in Y$ corresponds $h_{y}: \mathbf{C}^{*} \rightarrow \mathscr{T}, h_{y}(\zeta)=y \otimes \zeta$.

Now $W_{0}$ acts on $\mathscr{T}$ by $w: y \otimes \zeta \rightarrow w(y) \otimes \zeta$ and on $\mathscr{T} \times \mathbf{C}^{*}$ by $\left(t, \zeta_{1}\right) \rightarrow$ $\left(w(t), \zeta_{1}\right)$. This induces on functions the action of $W_{0}$ on $\mathscr{O}$ and $K$ considered in 3.12. We have

(a) $s_{\alpha}(t)=t h_{\check{\alpha}}\left(\theta_{\alpha}(t)\right)^{-1} \quad(\alpha \in R, t \in \mathscr{T})$.

We note also that if $\alpha \in R$, then

(b)

$$
\operatorname{ker}\left(h_{\check{\alpha}}: \mathbf{C}^{*} \rightarrow \mathscr{T}\right)= \begin{cases}\{1\}, & \text { if } \check{\alpha} \notin 2 Y, \\ \{1,-1\}, & \text { if } \check{\alpha} \in 2 Y .\end{cases}
$$

Lemma 3.15. Let $t \in \mathscr{T}$. Then $t$ is $W_{0}$-invariant if and only if for any $\alpha \in \Pi$ we have

(a)

$$
\begin{cases}\theta_{\alpha}(t)=1, & \text { if } \check{\alpha} \notin 2 Y, \\ \theta_{\alpha}(t)= \pm 1, & \text { if } \check{\alpha} \in 2 Y .\end{cases}
$$

Proof. Condition (a) is equivalent to $s_{\alpha}(t)=t$ (by 3.14(a) and (b)), and it remains to use the fact that $W_{0}$ is generated by $\left\{s_{\alpha} \mid \alpha \in \Pi\right\}$.

\section{The GRaded Hecke Algebra}

4.1. We assume that a parameter set for the root system $(X, Y, R, \check{R}, \Pi)$ has been fixed. In addition, we assume given a $W_{0}$-invariant element $t_{0} \in \mathscr{T}$.

To $t_{0}$ corresponds a $\mathbf{C}$-algebra homomorphism $h: \mathscr{O} \rightarrow \mathbf{C}$ defined by $h(v)$ $=1, h\left(\theta_{x}\right)=\theta_{x}\left(t_{0}\right) \quad(x \in X)$. By Lemma 3.15, we have for any $\alpha \in \Pi$,

(a)

$$
h\left(\theta_{\alpha}\right)= \begin{cases}1, & \text { if } \check{\alpha} \notin 2 Y, \\ \pm 1, & \text { if } \check{\alpha} \in 2 Y .\end{cases}
$$

Let $I$ be the kernel of $h$ (a maximal ideal of $\mathscr{O}$ ). Let $\overline{\mathscr{O}}^{i}=I^{i} / I^{i+1} \quad(i \geq 0)$, and let $\overline{\mathscr{O}}=\bigoplus_{i \geq 0} \overline{\mathscr{O}}^{i}$. This is a commutative graded $\mathbf{C}$-algebra in a natural way. The action of $W_{0}$ on $\mathscr{O}$ induces an action of $W_{0}$ on $\mathscr{\mathscr { O }}$ since $I$ is $W_{0^{-}}$ stable (recall that $t_{0}$ is $W_{0}$-invarant). For any $f \in \mathscr{O}$, we denote by $d(f)$ the image of $f-h(f) 1$ in $I / I^{2}=\overline{\mathscr{O}}^{1}$. We have

(b) $d\left(f f^{\prime}\right)=h(f) d\left(f^{\prime}\right)+h\left(f^{\prime}\right) d(f)$ for $f, f^{\prime} \in \mathscr{O}$.

If we regard $H$ as a left $\mathscr{O}$-module (see 3.12(b)), we can consider the filtration

(c) $H \supset I H \supset I^{2} H \supset \cdots$.

Lemma 4.2. The filtration $4.1(\mathrm{c})$ is compatible with the multiplication in $H$, i.e., $I^{i} H \cdot I^{j} H \subset I^{i+j} H \quad(i, j \geq 0)$. 
Proof. We first show

(a) $T_{w} \cdot I^{j} \subset I^{j} H$ for $j \geq 0, w \in W_{0}$.

We use induction on $l(w)$. We see that it is enough to consider the case where $w=s_{\alpha}(\alpha \in \Pi)$. We can also assume that $j \geq 1$. Using 3.12(d), we see that it is enough to check that for $f \in I^{j}$, we have

(b) $s_{\alpha}(f) \in I^{j}$.

(c)

$$
\frac{f-s_{\alpha}(f)}{\theta_{\alpha}^{c}-1} \in I^{j-1}, \quad \text { where } c= \begin{cases}1, & \text { if } \check{\alpha} \notin 2 Y, \\ 2, & \text { if } \check{\alpha} \in 2 Y .\end{cases}
$$

(d) $\theta_{\alpha} v^{2 \lambda(\alpha)}-1 \in I$ if $\check{\alpha} \notin 2 Y$.

(e) $\theta_{\alpha} v^{\lambda(\alpha)+h\left(\theta_{\alpha}\right) \lambda^{*}(\alpha)}-h\left(\theta_{\alpha}\right) \in I$ if $\check{\alpha} \in 2 Y$.

(Recall that if $\check{\alpha} \in 2 Y$, we have $h\left(\theta_{\alpha}\right)= \pm 1$ and that the expression in (e) is one of the two factors of the numerator of $\mathscr{G}(\alpha)$ in 3.8.) Now (b), (d), and (e) are obvious. To verify $(\mathrm{c})$, we note that

$$
\frac{f^{\prime} f^{\prime \prime}-s_{\alpha}\left(f^{\prime} f^{\prime \prime}\right)}{\theta_{\alpha}^{c}-1}=f^{\prime} \frac{f^{\prime \prime}-s_{\alpha}\left(f^{\prime \prime}\right)}{\theta_{\alpha}^{c}-1}+\frac{f^{\prime}-s_{\alpha}\left(f^{\prime}\right)}{\theta_{\alpha}^{c}-1} s_{\alpha}\left(f^{\prime \prime}\right)
$$

which reduces us to the case where $j=1$. We have for any $f \in \mathscr{O}$,

$$
\left(f-s_{\alpha}(f)\right) /\left(\theta_{\alpha}^{c}-1\right) \in \mathscr{O}
$$

(see 3.5). Thus (a) is proved. It implies $H \cdot I^{j} \subset I^{j} H$, where, in $H I^{j}, H$ is regarded as a right $\mathscr{O}$-module. Similarly, we see that $I^{j} H \subset H \cdot I^{j}$. Hence,

(f) $I^{j} H=H I^{j}$.

We have, using (f),

The lemma is proved.

$$
I^{i} H \cdot I^{j} H \subset I^{i} I^{j} H H=I^{i+j} H .
$$

4.3. From the previous lemma, we see that

(a) $\bar{H}=\bigoplus_{i>0} \bar{H}^{i}$, where $\bar{H}^{i}=I^{i} H / I^{i+1} H$

inherits from $H$ an associative $\mathrm{C}$-algebra structure (the graded algebra associated to the filtration 4.1(c)). Moreover, $\overline{\mathscr{O}}$ is naturally a subalgebra of $\bar{H}$. (From 3.12(b), we see that $I^{i} \cap I^{i+1} H=I^{i+1}$.)

Let $t_{w}$ be the image of $T_{w}$ in $H / I H=\bar{H}^{0} \quad\left(w \in W_{0}\right)$, and let $r=d(v) \in \overline{\mathscr{O}}^{1}$ (see 4.1). Then $t_{1}$ is the unit element of $\bar{H}$. Let $\bar{K}$ be the quotient field of क.

Proposition 4.4. We have

(a) $\bar{H}=\bigoplus_{w \in W_{0}} \overline{\mathscr{O}} \cdot t_{w}=\bigoplus_{w \in W_{0}} t_{w} \cdot \overline{\mathscr{O}}$.

(b) $t_{w^{\prime}} t_{w^{\prime}}=t_{w w^{\prime}}\left(w, w^{\prime} \in W_{0}\right)$.

(c) $\phi \cdot t_{s_{\alpha}}-t_{s_{\alpha}} s_{\alpha}(\phi)=\left(\phi-s_{\alpha}(\phi)\right)(g(\alpha)-1)(\forall \phi \in \overline{\mathscr{O}}, \alpha \in \Pi)$, where

$$
g(\alpha)=\left\{\begin{array}{ll}
\frac{d\left(\theta_{\alpha}\right)+2 \lambda(\alpha) r}{d\left(\theta_{\alpha}\right)}, & \text { if } \alpha \notin 2 Y, \\
\frac{d\left(\theta_{\alpha}\right)+\left(\lambda(\alpha)+h\left(\theta_{\alpha}\right) \lambda^{*}(\alpha)\right) r}{d\left(\theta_{\alpha}\right)}, & \text { if } \check{\alpha} \in 2 Y,
\end{array} \quad(g(\alpha) \in \bar{K}) .\right.
$$


Proof. (a) follows easily from definitions and from 3.12(b). To prove (b) we may assume that $w=s_{\alpha}(\alpha \in \Pi)$. We have

$$
T_{s_{\alpha}} T_{w^{\prime}}=\left\{\begin{array}{l}
T_{s_{\alpha} w^{\prime}}, \quad \text { if } l\left(s_{\alpha} w^{\prime}\right)=l\left(w^{\prime}\right)+1, \\
T_{s_{\alpha} w^{\prime}}+\left(v^{2 \lambda(\alpha)}-1\right)\left(T_{w^{\prime}}+T_{s_{\alpha} w^{\prime}}\right), \quad \text { if } l\left(s_{\alpha} w^{\prime}\right)=l\left(w^{\prime}\right)-1 .
\end{array}\right.
$$

Now $v^{2 \lambda(\alpha)}-1 \in I$. Taking images under $H \rightarrow \bar{H}^{0}$, we find (b). To prove (c), we note that $\overline{\mathscr{O}}$ is generated as a $\mathbf{C}$-algebra by $\overline{\mathscr{O}}^{1}$ and that if (c) is true for $\phi$ and $\phi^{\prime}$ in $\overline{\mathscr{O}}$, then it is also true for $\phi \phi^{\prime}$. Thus, it is enough to check (c) for $\phi \in \overrightarrow{\mathscr{O}}^{0}$ or $\overline{\mathscr{O}}^{1}$. For $\phi \in \overrightarrow{\mathscr{O}}^{0}$, (c) is obvious. If $\phi \in \overline{\mathscr{O}}^{1}$, we can write $\phi=d(f)$ for some $f \in I$. Apply $I H \rightarrow I H / I^{2} H=\bar{H}^{1}$ to the identity

(d) $f T_{s_{\alpha}}-T_{s_{\alpha}} s_{\alpha}(f)=\left(f-s_{\alpha}(f)\right)(\mathscr{G}(\alpha)-1)$.

The left-hand side of (d) is mapped to $\phi t_{s_{\alpha}}-t_{s_{\alpha}} s_{\alpha}(f)$, and it remains to show that the right-hand side of (d) (an element of $\mathscr{O} \cap I H=I$ ) satisfies

(e) $d\left(\left(f-s_{\alpha}(f)\right)(\mathscr{G}(\alpha)-1)\right)=\left(\phi-s_{\alpha}(\phi)\right)(g(\alpha)-1)$.

Let $\tilde{f}=\left(f-s_{\alpha}(f)\right) /\left(\theta_{d}^{c}-1\right) \quad(c$ as in 4.2(c)). From 4.2(c) we see that $\tilde{f} \in \mathscr{O}$. By 4.1(b), we have

$$
\begin{aligned}
\phi-s_{\alpha}(\phi) & =d\left(f-s_{\alpha}(f)\right)=d\left(\tilde{f}\left(\theta_{\alpha}^{c}-1\right)\right) \\
& =h(\tilde{f}) d\left(\theta_{\alpha}^{c}-1\right)+d(\tilde{f}) h\left(\theta_{\alpha}^{c}-1\right) \\
& =h(\tilde{f}) d\left(\theta_{\alpha}^{c}\right)=\operatorname{ch}(\tilde{f}) d\left(\theta_{\alpha}\right) .
\end{aligned}
$$

Hence,

(f)

$$
\begin{aligned}
\left(\phi-s_{\alpha}(\phi)\right)(g(\alpha)-1) & =\left(\phi-s_{\alpha}(\phi)\right) \frac{n r}{d \theta_{\alpha}} \\
& =\operatorname{cnh}(\tilde{f}) r=\operatorname{cnh}(\tilde{f}) d(v-1)=d(c n \tilde{f}(v-1)),
\end{aligned}
$$

where

$$
n=\left\{\begin{array}{l}
2 \lambda(\alpha), \quad \text { if } \check{\alpha} \notin 2 Y, \\
\lambda(\alpha)+h\left(\theta_{\alpha}\right) \lambda^{*}(\alpha), \quad \text { if } \check{\alpha} \in 2 Y .
\end{array}\right.
$$

Now $n \tilde{f}(v-1) \in I$, and from (f) we see that (e) is equivalent to the following statement:

(g) $\left(f-s_{\alpha}(f)\right)(\mathscr{G}(\alpha)-1)-c n \tilde{f}(v-1) \in I^{2}$.

Since $f-s_{\alpha}(f)=\tilde{f}\left(\theta_{\alpha}^{c}-1\right)$ and $\tilde{f} \in \mathscr{O}$, we see that (g) would be a consequence of the following statement:

(h) $\left(\theta_{\alpha}^{c}-1\right) \cdot(\mathscr{G}(\alpha)-1)-c n(v-1) \in I^{2}$.

Assume first that $\check{\alpha} \notin 2 Y$. Then the left-hand side of (h) is

$$
\theta_{\alpha}\left(v^{n}-1\right)-n(v-1)=\left(\theta_{\alpha}-1\right)\left(v^{n}-1\right)+\left(\frac{v^{n}-1}{v-1}-n\right)(v-1) \in I^{2} .
$$


Assume next that $\check{\alpha} \in 2 Y$. Then the left-hand side of $(\mathrm{h})$ is

$$
\begin{aligned}
& \theta_{2 \alpha}\left(v^{2 \lambda(\alpha)}-1\right)+\theta_{\alpha}\left(v^{\lambda(\alpha)+\lambda^{*}(\alpha)}-v^{\lambda(\alpha)-\lambda^{*}(\alpha)}\right)-2 n(v-1) \\
&=\left(\theta_{2 \alpha}-1\right)\left(v^{2 \lambda(\alpha)}-1\right)+\left(\theta_{\alpha}-h\left(\theta_{\alpha}\right)\right)\left(v^{\lambda(\alpha)+\lambda^{*}(\alpha)}-v^{\lambda(\alpha)-\lambda^{*}(\alpha)}\right) \\
&+\left(\frac{v^{2 \lambda(\alpha)}-1}{v-1}-2 \lambda(\alpha)\right)(v-1) \\
&+h\left(\theta_{\alpha}\right)\left(\frac{v^{\lambda(\alpha)+\lambda^{*}(\alpha)}-1}{v-1}-\left(\lambda(\alpha)+\lambda^{*}(\alpha)\right)\right)(v-1) \\
&-h\left(\theta_{\alpha}\right)\left(\frac{v^{\lambda(\alpha)-\lambda^{*}(\alpha)}-1}{v-1}-\left(\lambda(\alpha)-\lambda^{*}(\alpha)\right)\right)(v-1) \in I^{2},
\end{aligned}
$$

since $2 n=2 \lambda(\alpha)+h\left(\theta_{\alpha}\right)\left(\lambda(\alpha)+\lambda^{*}(\alpha)\right)-h\left(\theta_{\alpha}\right)\left(\lambda(\alpha)-\lambda^{*}(\alpha)\right)$. Hence, (h) is verified and the proposition is proved.

Proposition 4.5. Let $\overline{\mathscr{Z}}$ be the center of $\bar{H}$. Then $\overline{\mathscr{Z}}=\overline{\mathscr{O}}^{W_{0}} \quad\left(W_{0}\right.$-invariants in $\overline{\mathscr{O}})$.

The proof is the same as that of $[5,6.5]$.

4.6. Let $\bar{F}$ be the quotient field of $\overline{\mathscr{Z}}$. As in $3.12(\mathrm{a})$, we have

(a) $\overline{\mathscr{O}} \otimes_{\bar{X}} \bar{F} \stackrel{\approx}{\rightarrow} \bar{K}$.

Let $\bar{H}_{\bar{F}}$ be the $\bar{F}$-algebra $\bar{H} \otimes_{\bar{Z}} \bar{F}$. It contains $\bar{H}$ as a $\overline{\mathscr{Z}}$-subalgebra. We identify the subspace $\overline{\mathscr{O}} \otimes_{\bar{Z}} \bar{F}$ of $\bar{H}_{\bar{F}}$ with $\bar{K}$ using (a). Thus, we have $\bar{K} \subset$ $\bar{H}_{\bar{F}}$.

Tensoring 4.4(a) with $\bar{F}$ over $\overline{\mathscr{Z}}$, we obtain two decompositions

(b) $\bar{H}_{\bar{F}}=\bigoplus_{w \in W_{0}} t_{w} \cdot \bar{K}=\bigoplus_{w \in W_{0}} \bar{K} \cdot t_{w}$.

In the $\bar{F}$-algebra $\bar{H}_{\bar{F}}$, we have

(c) $\phi\left(t_{s_{\alpha}}+1\right)-\left(t_{s_{\alpha}}+1\right) s_{\alpha}(\phi)=\left(\phi-s_{\alpha}(\phi)\right) g(\alpha) \quad(\phi \in \bar{K}, \alpha \in \Pi)$.

(Note that the $W_{0}$-action on $\overline{\mathscr{Q}}$ extends to a $W_{0}$-action on $\bar{K}$ by field automorphisms.) This is deduced from Proposition 4.4(c) in the same way that $3.12(\mathrm{~d})$ is deduced from 3.9 .

\section{The Elements $\tau_{w}$ AND $\bar{\tau}_{w}$}

5.1. We preserve the setup of $\S \S 3$ and 4. For any $\alpha \in \Pi$, we define $\tau^{\alpha} \in H_{F}$, $\bar{\tau}^{\alpha} \in \bar{H}_{\bar{F}}$ by
(a) $\tau^{\alpha}+1=\left(T_{s_{\alpha}}+1\right) \mathscr{G}(\alpha)^{-1}, \bar{\tau}^{\alpha}+1=\left(t_{s_{\alpha}}+1\right) g(\alpha)^{-1}$.

Proposition 5.2. (a) There is a unique homomorphism $\tau: W_{0} \rightarrow$ (group of units of $H_{F}$ ) (resp. $\bar{\tau}: W_{0} \rightarrow\left(\right.$ group of units of $\left.\bar{H}_{\bar{F}}\right)$ ) such that $s_{\alpha} \rightarrow \tau^{\alpha}$ (resp. $s_{\alpha} \rightarrow$ $\left.\bar{\tau}^{\alpha}\right)$ for all $\alpha \in \Pi$.

(b) Let $\tau_{w}=\tau(w), \bar{\tau}_{w}=\bar{\tau}(w) \quad\left(w \in W_{0}\right)$. For any $f \in K($ resp. $\phi \in \bar{K})$, we have $f \tau_{w}=\tau_{w} w^{-1}(f)$ in $H_{F}$ (resp. $\phi \bar{\tau}_{w}=\bar{\tau}_{w} w^{-1}(\phi)$ in $\left.\bar{H}_{\bar{F}}\right)$.

The proof is based on the following lemma. 
Lemma 5.3. (a) Let $\Phi=\sum_{w \in W_{0}}(-1)^{l(w)} v^{-2 L(w)} T_{w} \in H$ and let $h \in H_{F}$ be such that $\Phi$ fh $=0$ (in $H_{F}$ ) for all $f \in \mathscr{O}$. Then $h=0$.

(b) Let $\bar{\Phi}=\sum_{w \in W_{0}}(-1)^{l(w)} t_{w} \in \bar{H}$ and let $\bar{h} \in \bar{H}_{\bar{F}}$ be such that $\bar{\Phi} \phi \bar{h}=0$ (in $\bar{H}_{\bar{F}}$ ) for all $\phi \in \overline{\mathscr{O}}$. Then $\bar{h}=0$.

Proof. Multiplying $h$ (resp. $\bar{h}$ ) by a suitable element of $\mathscr{Z}$ (resp. $\overline{\mathscr{Z}}$ ), we may assume that $h \in H$ (resp. $\bar{h} \in \bar{H}$ ). We shall assume statements (c) and (d) below:

(c) If $h \in H$ satisfies $\Phi f h \in(v-1) H$ for all $f \in \mathscr{O}$, then $h \in(v-1) H$.

(d) If $\bar{h} \in \bar{H}$ satisfies $\bar{\Phi} \phi \bar{h} \in r \bar{H}$ for all $\phi \in \overline{\mathscr{O}}$, then $\bar{h} \in r \bar{H}$.

Using (c) and (d) and the assumptions of (a) and (b), we see that $h=(v-1) h^{\prime}$ (resp. $\bar{h}=r \bar{h}^{\prime}$ ) for some $h^{\prime} \in H$ (resp. $\left.\bar{h}^{\prime} \in \bar{H}\right)$. But then $(v-1) \Phi f h^{\prime}=0$ for all $f \in \mathscr{O}$ (resp. $r \bar{\Phi} \phi \bar{h}^{\prime}=0$ for all $\phi \in \overline{\mathscr{O}}$ ). Hence, $\Phi f h^{\prime}=0$ for all $f \in \mathscr{O}$ (resp. $\bar{\Phi} \phi \bar{h}^{\prime}=0$ for all $\phi \in \overline{\mathscr{O}}$ ), and using (c) and (d) again, we see that $h^{\prime} \in(v-1) H$ (resp. $\bar{h}^{\prime} \in r \bar{H}$ ), so that $h \in(v-1)^{2} H$ (resp. $\bar{h} \in r^{2} \bar{H}$ ). Continuing in this way, we see that $h \in(v-1)^{i} H$ (resp. $\bar{h} \in r^{i} \bar{H}$ ) for all $i \geq 1$. Hence, $h=0$ and $\bar{h}=0$.

It remains to prove (c) and (d).

First note that

$$
f T_{s_{\alpha}}-T_{s_{\alpha}} s_{\alpha}(f) \in(v-1) H, \quad \phi t_{s_{\alpha}}-t_{s_{\alpha}} s_{\alpha}(\phi) \in r \bar{H}
$$

for all $f \in \mathscr{O}, \alpha \in \Pi, \phi \in \overline{\mathscr{O}}$ (see Proposition 3.6 and Proposition 4.4(c)). Hence, by induction on $l\left(w^{\prime}\right)$, we have

(e)

$$
\left\{\begin{array}{l}
f T_{w^{\prime}}-T_{w^{\prime}} w^{\prime-1}(f) \in(v-1) H, \\
\phi t_{w^{\prime}}-t_{w^{\prime}} w^{\prime-1}(\phi) \in r \bar{H}
\end{array}\right.
$$

for all $w^{\prime} \in W_{0}$.

Let $h, \bar{h}$ be as in (c) and (d).

We can write uniquely $h=\sum_{w^{\prime} \in W_{0}} T_{w^{\prime}} f_{w^{\prime}}, \bar{h}=\sum_{w^{\prime} \in W_{0}} t_{w^{\prime}} \phi_{w^{\prime}} \quad\left(f_{w^{\prime}} \in \mathscr{O}\right.$, $\left.\phi_{w^{\prime}} \in \overline{\mathscr{O}}\right)$. See 3.12(b) and Proposition 4.4(a). By assumption, we have

$$
\begin{gathered}
\sum_{w^{\prime}} \Phi f T_{w^{\prime}} f_{w^{\prime}} \in(v-1) H \quad(\forall f \in \mathscr{O}), \\
\sum_{w^{\prime}} \bar{\Phi} \phi t_{w^{\prime}} \phi_{w^{\prime}} \in r \bar{H} \quad(\forall \phi \in \overline{\mathscr{O}}) .
\end{gathered}
$$

Hence, using (e), we have

$$
\begin{gathered}
\sum_{w^{\prime}} \Phi T_{w^{\prime}} w^{\prime^{-1}}(f) f_{w^{\prime}} \in(v-1) H \quad(\forall f \in \mathscr{O}), \\
\sum \bar{\Phi} t_{w^{\prime}} w^{-1}(\phi) \phi_{w^{\prime}} \in r \bar{H} \quad(\forall \phi \in \overline{\mathscr{O}}) .
\end{gathered}
$$


Now $T_{w} T_{w^{\prime}}-T_{w w^{\prime}} \in(v-1) H, v^{-2 L(w)}-1 \in(v-1) H$ for all $w, w^{\prime} \in W$. Hence, we deduce

$$
\begin{gathered}
\sum_{w, w^{\prime}}(-1)^{l(w)} T_{w w^{\prime}} w^{\prime^{-1}}(f) f_{w^{\prime}} \in(v-1) H \quad(\forall f \in \mathscr{O}), \\
\sum_{w, w^{\prime}}(-1)^{l(w)} t_{w w^{\prime}} w^{-1}(\phi) \phi_{w^{\prime}} \in r \bar{H} \quad(\forall \phi \in \overline{\mathscr{O}}),
\end{gathered}
$$

or, setting $w w^{\prime}=w^{\prime \prime}$,

$$
\begin{gathered}
\sum_{w^{\prime}, w^{\prime \prime}}(-1)^{l\left(w^{\prime} w^{\prime \prime}\right)} T_{w^{\prime \prime}} w^{\prime^{-1}}(f) f_{w^{\prime}} \in(v-1) H \quad(\forall f \in \mathscr{O}), \\
\sum_{w^{\prime}, w^{\prime \prime}}(-1)^{l\left(w^{\prime} w^{\prime \prime}\right)} t_{w^{\prime \prime}} w^{\prime^{-1}}(\phi) \phi_{w^{\prime}} \in r \bar{H} \quad(\forall \phi \in \overline{\mathscr{O}}) .
\end{gathered}
$$

Using 3.12(b) and Proposition 4.4(a), we deduce that

(f) $\sum_{w^{\prime}}(-1)^{l\left(w^{\prime}\right)} w^{\prime^{-1}}(f) f_{w^{\prime}} \in(v-1) \mathscr{O}(\forall f \in \mathscr{O})$,

(g) $\sum_{w^{\prime}}(-1)^{l\left(w^{\prime}\right)} w^{\prime^{-1}}(\phi) \phi_{w^{\prime}} \in r \overline{\mathscr{O}} \quad(\forall \phi \in \overline{\mathscr{O}})$.

We write (f) (resp. (g)) for $f=\theta_{x}^{i}, i=0,1, \ldots,\left|W_{0}\right|-1$ (resp. $\phi=\phi_{1}^{i}$, $i=0,1, \ldots,\left|W_{0}\right|-1$ ), where $x \in X$ (resp. $\phi_{1} \in \overline{\mathscr{O}}^{1}$ ) is fixed such that $x \neq w(x)$ (resp. $\phi_{1} \neq w\left(\phi_{1}\right)$ ) for all $w \in W_{0}$. Using Cramer's rule, we see that for all $w^{\prime} \in W_{0}$, we have

(h) $\delta f_{w^{\prime}} \in(v-1) \mathscr{O}$ (resp. $\left.\bar{\delta} \phi_{w^{\prime}} \in r \overline{\mathscr{O}}\right)$,

where $\delta$ is a Vandermonde determinant in the variables $w^{-1}\left(\theta_{x}\right), w \in W_{0}$ (resp. $w^{-1}\left(\phi_{1}\right), w \in W_{0}$ ). Hence, $\delta$ (resp. $\bar{\delta}$ ) is a product of elements of $\mathscr{O}$ (resp. $\overline{\mathscr{O}}$ ) of the form $w_{1}^{-1}\left(\theta_{x}\right)-w_{2}^{-1}\left(\theta_{x}\right), w_{1} \neq w_{2}$ (resp. $w_{1}^{-1}\left(\phi_{1}\right)-w_{2}^{-1}\left(\phi_{1}\right)$, $w_{1} \neq w_{2}$ ). These factors of $\delta$ (resp. $\delta$ ) are nonzero and are not divisible by $v-1$ (resp. $r$ ). Since $\mathscr{O}$ (resp. $\overline{\mathscr{O}}$ ) is a unique factorization domain, from (h) it follows that $f_{w^{\prime}} \in(v-1) \mathscr{O}$ (resp. $\phi_{w^{\prime}} \in r \overline{\mathscr{O}}$ ) for all $w^{\prime} \in W_{0}$, and (c) and (d) follow. The lemma is proved.

5.4. Proof of Proposition 5.2. If $\Phi$ is as in 4.3(a), we have clearly

(a) $\Phi\left(T_{s_{\alpha}}+1\right)=0$ for any $\alpha \in \Pi$.

If $f \in K, \alpha \in \Pi$, we have (in $H_{F}$ )

$$
\begin{aligned}
\Phi f\left(\tau^{\alpha}+1\right) & =\Phi f\left(T_{s_{\alpha}}+1\right) \mathscr{G}(\alpha)^{-1} \\
& =\Phi\left(\left(T_{s_{\alpha}}+1\right) s_{\alpha}(f)+\left(f-s_{\alpha}(f)\right) \mathscr{G}(\alpha)\right) \mathscr{G}(\alpha)^{-1} \quad(\text { see 3.12(d)) } \\
& =\Phi\left(f-s_{\alpha}(f)\right) \quad(\text { see }(\mathrm{a})) .
\end{aligned}
$$

Hence, $\Phi f \tau^{\alpha}=-\Phi s_{\alpha}(f)$.

Applying this identity repeatedly, we get

(b) $\Phi f \tau^{\alpha_{1}} \tau^{\alpha_{2}} \cdots \tau^{\alpha_{p}}=(-1)^{p} \Phi s_{\alpha_{p}} \cdots s_{\alpha_{2}} s_{\alpha_{1}}(f)$

for any sequence $\alpha_{1}, \alpha_{2}, \ldots, \alpha_{p}$ in $\Pi$. Assume now that $s_{\alpha_{1}} s_{\alpha_{2}} \cdots s_{\alpha_{p}}=1$ in $W_{0}$. Then $p$ must be even and we also have $s_{\alpha_{p}} \cdots s_{\alpha_{2}} s_{\alpha_{1}}=1$ in $W_{0}$. From 
(b), we deduce that $\tau^{\alpha_{1}} \tau^{\alpha_{2}} \cdots \tau^{\alpha_{p}}=1$. This proves Proposition 5.2(a) for $\tau$. We now rewrite 3.12(d) using 5.1(a):

$$
f\left(\tau^{\alpha}+1\right) \mathscr{G}(\alpha)-\left(\tau^{\alpha}+1\right) \mathscr{G}(\alpha) s_{\alpha}(f)=\left(f-s_{\alpha}(f)\right) \mathscr{G}(\alpha) .
$$

Cancelling $\mathscr{G}(\alpha)$, we obtain $f \tau^{\alpha}=\tau^{\alpha} s_{\alpha}(f)(f \in K, \alpha \in \Pi)$ and Proposition 5.2(b) follows (for $\tau$ ). The proof for $\bar{\tau}$ is completely similar.

Proposition 5.5. (a) $H_{F}=\bigoplus_{w \in W_{0}} \tau_{w} \cdot K=\bigoplus_{w \in W_{0}} K \cdot \tau_{w}$.

(b) $\bar{H}_{\bar{F}}=\bigoplus_{w \in W_{0}} \bar{\tau}_{w} \bar{K}=\bigoplus_{w \in W_{0}} \bar{K} \bar{\tau}_{w}$.

Proof. Let $H_{F}^{\prime}=\sum_{w \in W_{0}} \tau_{w} \cdot K$. We show by induction on $l\left(w^{\prime}\right)$ that $T_{w^{\prime}} \subset H_{F}^{\prime}$ for any $w^{\prime} \in W_{0}$. It is enough to show that $T_{s_{\alpha}} H_{F}^{\prime} \subset H_{F}^{\prime}$ for any $\alpha \in \Pi$ or, using 5.1(a), that $\tau^{\alpha} H_{F}^{\prime} \subset H_{F}^{\prime}$ and $K H_{F}^{\prime} \subset H_{F}^{\prime}$. Now $\tau^{\alpha} H_{F}^{\prime} \subset H_{F}^{\prime}$ follows from the definition of $\tau_{w}$ and $K H_{F}^{\prime} \subset H_{F}^{\prime}$ follows from Proposition 5.2(b). Thus, $T_{w^{\prime}} \subset H_{F}^{\prime}$ for all $w^{\prime} \in W_{0}$. Hence, $\sum_{w^{\prime}} T_{w^{\prime}} K \subset H_{F}^{\prime}$. Hence $H_{F}^{\prime}=H_{F}$ (see 3.12(c)). Now $T_{w}\left(w \in W_{0}\right)$ form a basis of $H_{F}$ as a right $K$-vector space, and we have just seen that $\tau_{w}\left(w \in W_{0}\right)$ form a set of generators of $H_{F}$ as a right $K$-vector space. It follows that $\tau_{w}\left(w \in W_{0}\right)$ form a basis of this vector space. Hence, $H_{F}=\bigoplus_{w \in W_{0}} \tau_{w} \cdot K$. The equality $H_{F}=\bigoplus_{w \in W_{0}} K \cdot \tau_{w}$ and the equalities in (b) are proved similarly.

\section{SOME BRAID GROUP RELATIONS}

6.1. This section contains a result which is needed in the proof of Theorem 8.6. We fix $\alpha \neq \beta$ in $\Pi$. Let $W_{0}^{\alpha \beta}$ be the subgroup of $W_{0}$ generated by $s_{\alpha}$, $s_{\beta}$, and let $m$ be the order of the product $s_{\alpha} s_{\beta}$. We assume given a finite set $\mathscr{P}$ on which $W_{0}^{\alpha \beta}$ acts by permutations and an element $c \in \mathscr{P}$. We define elements $\xi_{1}, \xi_{2}, \ldots, \eta_{1}, \eta_{2}, \ldots$ in $H_{F}$ by

$$
\begin{aligned}
& \xi_{1}= \begin{cases}T_{s_{\alpha}}, & \text { if } s_{\alpha}(c)=c, \\
\tau^{\alpha}, & \text { if } s_{\alpha}(c) \neq c,\end{cases} \\
& \eta_{1}=\left\{\begin{array}{cc}
T_{s_{\beta}}, & \text { if } s_{\beta}(c)=c, \\
\tau^{\beta}, & \text { if } s_{\beta}(c) \neq c,
\end{array}\right. \\
& \xi_{2}= \begin{cases}T_{s_{\beta}}, & \text { if } s_{\beta} s_{\alpha}(c)=s_{\alpha}(c), \\
\tau^{\beta}, & \text { if } s_{\beta} s_{\alpha}(c) \neq s_{\alpha}(c),\end{cases} \\
& \eta_{2}= \begin{cases}T_{s_{\alpha}}, & \text { if } s_{\alpha} s_{\beta}(c)=s_{\beta}(c), \\
\tau^{\alpha}, & \text { if } s_{\alpha} s_{\beta}(c) \neq s_{\beta}(c),\end{cases} \\
& \xi_{3}= \begin{cases}T_{s_{\alpha}}, & \text { if } s_{\alpha} s_{\beta} s_{\alpha}(c)=s_{\beta} s_{\alpha}(c), \\
\tau^{\alpha}, & \text { if } s_{\alpha} s_{\beta} s_{\alpha}(c) \neq s_{\beta} s_{\alpha}(c),\end{cases} \\
& \eta_{3}= \begin{cases}T_{s_{\beta}}, & \text { if } s_{\beta} s_{\alpha} s_{\beta}(c)=s_{\alpha} s_{\beta}(c), \\
\tau^{\beta}, & \text { if } s_{\beta} s_{\alpha} s_{\beta}(c) \neq s_{\alpha} s_{\beta}(c),\end{cases}
\end{aligned}
$$

etc.

Proposition 6.2. We have $\xi_{1} \xi_{2} \cdots \xi_{m}=\eta_{1} \eta_{2} \cdots \eta_{m}$ in $H_{F}$.

Proof. Let $Z$ be the set of reflections in $W_{0}^{\alpha \beta}$ which keep $c$ fixed.

To simplify notation, we set $\tau^{\alpha}=\tau, \tau^{\beta}=\tau^{\prime}, T_{s_{\alpha}}=T, T_{s_{\beta}}=T^{\prime}$. Assume first that $Z$ is empty. Then the identity to be proved is

(a) $\tau \tau^{\prime} \tau \cdots=\tau^{\prime} \tau \tau^{\prime} \ldots$ ( $m$ factors $)$.

This is known from Proposition 5.2(a). We denote by $\tilde{\tau}$ the two sides of (a). 
When $Z$ contains $m$ reflections, the identity to be proved is

(b) $T T^{\prime} T \cdots=T^{\prime} T T^{\prime} \cdots$

which follows from the definition of $H$.

Assume now that $Z=\left\{s_{\alpha}\right\}$. The identity to be proved is

(c) $T \tau^{\prime}=\tau^{\prime} T$ (if $m=2$ ), $T \tau^{\prime} \tau=\tau^{\prime} \tau T^{\prime}$ (if $m=3$ ), $T \tau^{\prime} \tau \tau^{\prime}=\tau^{\prime} \tau \tau^{\prime} T$ (if $m=4$ ), $T \tau^{\prime} \tau \tau^{\prime} \tau \tau^{\prime}=\tau^{\prime} \tau \tau^{\prime} \tau \tau^{\prime} T$ (if $m=6$ ).

A simple computation using Proposition 5.2(a) and (b) shows that both sides of (c) are equal to

$$
\begin{cases}\tilde{\tau}\left(1+\tau^{\beta}\left(1-\mathscr{G}(\beta)^{-1}\right)\right) \mathscr{G}(\beta), & \text { if } m=3, \\ \tilde{\tau}\left(1+\tau^{\alpha}\left(1-\mathscr{G}(\alpha)^{-1}\right)\right) \mathscr{G}(\alpha), & \text { if } m=2,4, \text { or } 6 .\end{cases}
$$

The case where $Z=\left\{s_{\beta}\right\}$ is entirely similar. We can assume from now on that $m \geq 3$. Assume that $Z=\left\{s_{\alpha} s_{\beta} s_{\alpha}\right\}$. The identity to be proved is

(d) $\tau T^{\prime} \tau=\tau^{\prime} T \tau^{\prime}$ (if $m=3$ ), $\tau T^{\prime} \tau \tau^{\prime}=\tau^{\prime} \tau T^{\prime} \tau$ (if $m=4$ ), $\tau T^{\prime} \tau \tau^{\prime} \tau \tau^{\prime}=$ $\tau^{\prime} \tau \tau^{\prime} \tau T^{\prime} \tau$ (if $m=6$ ).

This can be formally deduced from (c) using the fact that $\tau^{2}=\tau^{\prime^{2}}=1$. More generally, the case where $Z$ consists of a single reflection follows formally from (c). We can assume from now on that $m \geq 4$. When $m=4$, it remains to consider the cases where $Z=\left\{s_{\beta}, s_{\alpha} s_{\beta} s_{\alpha}\right\}$ and $Z=\left\{s_{\alpha}, s_{\beta} s_{\alpha} s_{\beta}\right\}$. We can assume that $\langle\alpha, \check{\beta}\rangle=-1,\langle\beta, \check{\alpha}\rangle=-2$. The identities to be proved are, respectively,

(e) $\tau T^{\prime} \tau T^{\prime}=T^{\prime} \tau T^{\prime} \tau, \tau^{\prime} T \tau^{\prime} T=T \tau^{\prime} T \tau^{\prime}$

A simple computation using Proposition 5.2(a) and (b) shows that both sides of the first identity of (e) are equal to

$$
\begin{aligned}
\tilde{\tau}\left(1+\tau^{\beta}\left(1-\mathscr{G}(\beta)^{-1}\right)+\tau^{\alpha} \tau^{\beta} \tau^{\alpha}\left(1-\mathscr{G}(2 \alpha+\beta)^{-1}\right)\right. \\
\left.\quad+\tilde{\tau}\left(1-\mathscr{G}(\beta)^{-1}\right)\left(1-\mathscr{G}(2 \alpha+\beta)^{-1}\right)\right) \mathscr{G}(\beta) \mathscr{G}(2 \alpha+\beta)
\end{aligned}
$$

and both sides of the second identity of (e) are equal to

$$
\begin{aligned}
\tilde{\tau}\left(1+\tau^{\alpha}\left(1-\mathscr{G}(\alpha)^{-1}\right)+\tau^{\beta} \tau^{\alpha} \tau^{\beta}\left(1-\mathscr{G}(\alpha+\beta)^{-1}\right)\right. \\
\left.+\tilde{\tau}\left(1-\mathscr{G}(\alpha)^{-1}\right)\left(1-\mathscr{G}(\alpha+\beta)^{-1}\right)\right) \mathscr{G}(\alpha) \mathscr{G}(\alpha+\beta) ;
\end{aligned}
$$

we have

$$
\begin{gathered}
\mathscr{G}(2 \alpha+\beta)=s_{\alpha}(\mathscr{G}(\beta)), \quad \mathscr{G}(\alpha+\beta)=s_{\beta}(\mathscr{G}(\alpha)), \\
s_{\beta}(\mathscr{G}(2 \alpha+\beta))=\mathscr{G}(2 \alpha+\beta), \quad s_{\alpha}(\mathscr{G}(\alpha+\beta))=\mathscr{G}(\alpha+\beta) \quad(\text { see 3.13). }
\end{gathered}
$$

When $m=6$, we can assume that $\langle\alpha, \check{\beta}\rangle=-1,\langle\beta, \check{\alpha}\rangle=-3$. It remains to consider the cases where

$$
\begin{gathered}
Z=\left\{s_{\alpha}, s_{\beta} s_{\alpha} s_{\beta} s_{\alpha} s_{\beta}\right\}, \quad Z=\left\{s_{\beta}, s_{\alpha} s_{\beta} s_{\alpha} s_{\beta} s_{\alpha}\right\}, \quad Z=\left\{s_{\alpha} s_{\beta} s_{\alpha}, s_{\beta} s_{\alpha} s_{\beta}\right\}, \\
Z=\left\{s_{\beta}, s_{\alpha} s_{\beta} s_{\alpha}, s_{\beta} s_{\alpha} s_{\beta} s_{\alpha} s_{\beta}\right\}, \quad Z=\left\{s_{\alpha}, s_{\beta} s_{\alpha} s_{\beta}, s_{\alpha} s_{\beta} s_{\alpha} s_{\beta} s_{\alpha}\right\} .
\end{gathered}
$$

The identities to be proved are, respectively,

(f) $T \tau^{\prime} \tau T^{\prime} \tau \tau^{\prime}=\tau^{\prime} \tau T^{\prime} \tau \tau^{\prime} T, \tau \tau^{\prime} T \tau^{\prime} \tau T^{\prime}=T^{\prime} \tau \tau^{\prime} T \tau^{\prime} \tau, \tau T^{\prime} \tau \tau^{\prime} T \tau^{\prime}=\tau^{\prime} T \tau^{\prime} \tau T^{\prime} \tau$, $\tau T^{\prime} \tau T^{\prime} \tau T^{\prime}=T^{\prime} \tau T^{\prime} \tau T^{\prime} \tau, \tau^{\prime} T \tau^{\prime} T \tau^{\prime} T=T \tau^{\prime} T \tau^{\prime} T \tau^{\prime}$. 
These can again be verified using Proposition 5.2(a) and (b). We omit the details. (Note only that the first three identities in (f) are formally equivalent to each other.)

\section{Completions}

\subsection{We preserve the setup of $\S \S 3$ and 4 .}

Let $\mathfrak{t}=Y \otimes \mathbf{C}$. Any element $x \in X$ may be regarded as a linear form $\mathfrak{t} \rightarrow \mathbf{C}$, $y \otimes z \rightarrow z\langle x, y\rangle$. This linear form will be denoted again by $x$. We shall denote by $\bar{x}$ the composition

$$
\mathfrak{t} \oplus \mathbf{C} \stackrel{\mathrm{pr}_{1}}{\rightarrow} \mathfrak{t} \stackrel{x}{\rightarrow} \mathbf{C} .
$$

There is a unique isomorphism $\operatorname{Hom}(\mathfrak{t} \oplus \mathbf{C}, \mathbf{C}) \stackrel{\approx}{\rightarrow} \overline{\mathscr{O}}^{1}=I / I^{2}$ (see 4.1) such that $\bar{x} \mapsto d\left(\theta_{x}\right), \mathrm{pr}_{2} \mapsto r$. We shall identify these two vector spaces. In particular, we shall identify $r$ with $\mathrm{pr}_{2}: t \otimes \mathbf{C} \rightarrow \mathbf{C}$. This identification is compatible with the natural $W_{0}$-actions. It follows that $\overline{\mathscr{O}}$ may be identified with the algebra of regular functions $\mathfrak{t} \oplus \mathbf{C} \rightarrow \mathbf{C}$.

7.2. By Proposition 3.11 (resp. Proposition 4.5), $\mathscr{Z}=\mathscr{O}^{W_{0}}\left(\right.$ resp. $\overline{\mathscr{Z}}=\overline{\mathscr{O}}^{W_{0}}$ ) may be identified with the coordinate ring of $\left(\mathscr{T} \times \mathbf{C}^{*}\right) / W_{0}=\left(\mathscr{T} / W_{0}\right) \times \mathbf{C}^{*}$ (resp. $\left.(t \oplus \mathbf{C}) / W_{0}=\left(t / W_{0}\right) \times \mathbf{C}\right)$ so that the inclusion $\mathscr{Z} \subset \mathscr{O}$ (resp. $\left.\overline{\mathscr{Z}} \subset \overline{\mathscr{O}}\right)$ corresponds to the orbit map $\mathscr{T} \times \mathbf{C}^{*} \rightarrow\left(\mathscr{T} \times \mathbf{C}^{*}\right) / W_{0}$ (resp. $\left.t \oplus \mathbf{C} \rightarrow(t \oplus \mathbf{C}) / W_{0}\right)$. Hence, the maximal ideals of $\mathscr{Z}$ (resp. $\overline{\mathscr{X}}$ ) are of the form

(a) $J_{\left(\Sigma, v_{0}\right)}=\left\{f \in \mathscr{Z} \mid f\left(t, v_{0}\right)=0, \forall t \in \Sigma\right\}$

(resp. $\bar{J}_{\left(\bar{\Sigma}, r_{0}\right)}=\left\{\phi \in \overline{\mathscr{Z}} \mid \phi\left(\xi, r_{0}\right)=0, \forall \xi \in \bar{\Sigma}\right\}$, where $\Sigma$ (resp. $\bar{\Sigma}$ ) is a $W_{0}$-orbit in $\mathscr{T}($ resp. $\mathfrak{t})$ and $v_{0} \in \mathbf{C}^{*}$ (resp. $\left.r_{0} \in \mathbf{C}\right)$.

7.3. We now fix $\left(\Sigma, v_{0}\right)$ (resp. $\left.\left(\bar{\Sigma}, r_{0}\right)\right)$ as in 7.2. We denote by $\widehat{\mathscr{Z}}$ (resp. $\widehat{\overline{\mathscr{Z}}})$ the $J_{\left(\Sigma, v_{0}\right)}$-adic (resp. $\bar{J}_{\left(\bar{\Sigma}, r_{0}\right)}$-adic) completion of $\mathscr{Z}$ (resp. $\overline{\mathscr{Z}}$ ). We define

$$
\widehat{\mathscr{O}}=\mathscr{O} \underset{\mathscr{Z}}{\otimes} \widehat{\mathscr{Z}}, \quad \hat{\mathscr{O}}=\overline{\mathscr{O}} \underset{\mathscr{X}}{\underset{\mathscr{X}}{\otimes}}, \quad \widehat{H}=H \underset{\mathscr{X}}{\otimes} \widehat{\mathscr{Z}}, \quad \hat{H}=\bar{H} \underset{\mathscr{X}}{\otimes} \widehat{\widehat{\mathscr{X}}}
$$

Then $\widehat{\mathscr{O}}, \hat{H}$ (resp. $\hat{\mathscr{O}}, \widehat{\bar{H}}$ ) are naturally $\widehat{\mathscr{Z}}$ - (resp. $\widehat{\mathscr{\mathscr { Z }}}$-) algebras and the imbeddings $\mathscr{Z} \subset \mathscr{O} \subset H$ (resp. $\overline{\mathscr{Z}} \subset \overline{\mathscr{O}} \subset \bar{H}$ ) give rise to imbeddings $\widehat{\mathscr{Z}} \subset$ $\widehat{\mathscr{O}} \subset \widehat{H}$ (resp. $\widehat{\mathscr{Z}} \subset \widehat{\hat{\mathscr{O}}} \subset \widehat{\bar{H}}$ ). We shall regard $\mathscr{O}$ and $H$ as $\mathscr{Z}$-subalgebras of $\hat{\mathscr{O}}$ and $\hat{H}$ in the obvious way. We also regard $\widehat{\mathscr{O}}$ and $\bar{H}$ as $\bar{Z}$-subalgebras of $\hat{\mathscr{O}}$ and $\hat{\bar{H}}$. We note that the $\mathscr{Z}$-linear (resp. $\bar{Z}$-linear) $W_{0}$-action on $\mathscr{O}$ (resp. $\overline{\mathscr{O}})$ extends to a $\widehat{\mathscr{Z}}$-linear (resp. $\widehat{\mathscr{Z}}$-linear) $W_{0}$-action on $\hat{\mathscr{O}}$ (resp. $\widehat{\mathscr{\mathscr { O }}}$ ), and we have

(a) $\widehat{\mathscr{Z}}=\widehat{\mathscr{O}}^{W_{0}}, \widehat{\mathscr{X}}^{=\hat{\mathscr{O}}^{W_{0}}}$.

Let $\widehat{F}$ (resp. $\hat{\bar{F}}$ ) be the quotient field of $\widehat{\mathscr{Z}}$ (resp. $\widehat{\mathscr{X}}$ ).

Let $\widehat{K}$ (resp. $\hat{\bar{K}}$ ) be the full ring of quotients of $\hat{\mathscr{O}}$ (resp. $\hat{\mathscr{O}}$ ). We have $\widehat{F} \subset \widehat{K}, \hat{\mathscr{O}} \subset \widehat{K}, \widehat{\bar{F}} \subset \hat{\bar{K}}, \hat{\overline{\mathscr{O}}} \subset \widehat{\bar{K}}$. 
Just as in 3.12(a), we see using (a) that

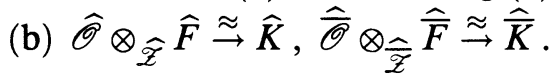

This is defined by multiplication in $\widehat{K}$ or $\widehat{\bar{K}}$. Using the definition of $\widehat{\mathscr{O}}$, we obtain $\bar{K} \cong \mathscr{O} \otimes_{\mathscr{Z}} \widehat{F} \cong \mathscr{O} \otimes_{\mathscr{X}} F \otimes_{F} \widehat{F}$. Hence,

(c) $\widehat{K} \cong K \otimes_{F} \widehat{F}$

(see 3.12(a)). Similarly,

(d) $\widehat{\bar{K}} \cong \bar{K} \otimes_{\bar{F}} \widehat{\bar{F}}$.

7.4. We consider the $\widehat{F}$-algebra $\widehat{H}_{F}=H \otimes_{\mathscr{X}} \widehat{F}=H_{F} \otimes_{F} \widehat{F}$ (resp. the $\widehat{\bar{F}}$ algebra $\hat{\bar{H}}_{\bar{F}}=\bar{H} \otimes_{\overline{\mathcal{X}}} \hat{\bar{F}}=\bar{H}_{\bar{F}} \otimes_{\bar{F}} \hat{\bar{F}}$ ). We can naturally regard $H_{F}$ (resp. $\bar{H}_{\bar{F}}$ ) as an $F$-subalgebra of $\widehat{H}_{F}$ (resp. $\bar{F}$-subalgebra of $\widehat{\bar{H}}_{\bar{F}}$ ). Moreover, the imbedding $K \subset H_{F}$ (see 3.12, resp. $\bar{K} \subset \bar{H}_{\bar{F}}$ (see 4.6)) induces an imbedding $\widehat{K}=K \otimes_{F} \widehat{F} \subset \widehat{H}_{F}$ (resp. $\widehat{\bar{K}}=\bar{K} \otimes_{F} \widehat{\bar{F}} \subset \widehat{\bar{H}}_{\bar{F}}$ ). From 3.12(c), 4.6(b), and Proposition 5.5, we deduce that

(a)

$$
\left\{\begin{array}{l}
\widehat{H}_{F}=\bigoplus \tau_{w} \widehat{K}=\oplus \widehat{K} \tau_{w}=\oplus T_{w} \widehat{K}=\oplus \widehat{K} \cdot T_{w}, \\
\widehat{\widehat{H}}_{\bar{F}}=\bigoplus \bar{\tau}_{w} \widehat{\bar{K}}=\bigoplus \widehat{\bar{K}} \bar{\tau}_{w}=\bigoplus t_{w} \widehat{\bar{K}}=\bigoplus \widehat{\bar{K}} t_{w} .
\end{array}\right.
$$

From 3.12(b) and Proposition 4.4(a), we deduce that

(b)

$$
\left\{\begin{array}{l}
\hat{H}=\oplus T_{w} \widehat{\mathscr{O}}=\bigoplus \widehat{\mathscr{O}} T_{w}, \\
\widehat{\hat{H}}=\bigoplus t_{w} \widehat{\mathscr{O}}=\bigoplus \widehat{\mathscr{O}} t_{w}
\end{array}\right.
$$

(all direct sums are taken over $w \in W_{0}$ ).

7.5. For any $t \in \Sigma$ and $\bar{t} \in \bar{\Sigma}$, we define

$$
\begin{aligned}
I_{(t)} & =\left\{f \in \mathscr{O} \mid f\left(t, v_{0}\right)=0\right\}, \quad \text { a maximal ideal of } \mathscr{O}, \\
\bar{I}_{(\bar{t})} & =\left\{\phi \in \overline{\mathscr{O}} \mid \phi\left(\bar{t}, r_{0}\right)=0\right\}, \quad \text { a maximal ideal of } \overline{\mathscr{O}}, \\
\widehat{\mathscr{O}}_{t} & =I_{(t)} \text {-adic completion of } \mathscr{O}, \\
\hat{\mathscr{O}}_{\bar{t}} & =\bar{I}_{(\bar{t})} \text {-adic completion of } \overline{\mathscr{O}} .
\end{aligned}
$$

We have $J_{\left(\Sigma, v_{0}\right)} \subset I_{(t)}$ (resp. $\left.\bar{J}_{\left(\bar{\Sigma}, r_{0}\right)} \subset I_{(\bar{t})}\right)$. Hence, the identity map $\mathscr{O} \rightarrow \mathscr{O}$ (resp. $\overline{\mathscr{O}} \rightarrow \overline{\mathscr{O}}$ ) extends continuously to a homomorphism of completions $\hat{\mathscr{O}} \rightarrow$ $\hat{\mathscr{O}}_{t}$ (resp. $\left.\hat{\mathscr{O}} \rightarrow \hat{\mathscr{O}}_{\bar{t}}\right)$. Taking the direct sum over $t$ (resp. $\bar{t}$ ), we obtain an isomorphism of $\widehat{\mathscr{Z}}$-algebras

(a) $\hat{\mathscr{O}} \approx \bigoplus_{t \in \Sigma} \hat{\mathscr{O}}_{t}$

(resp. an isomorphism of $\widehat{\mathscr{Z}}$-algebras

(b) $\hat{\mathscr{O}} \approx \bigoplus_{i \in \bar{\Sigma}} \hat{\overline{\mathscr{O}}}_{\bar{t}}$ ).

(In a direct sum of algebras, the product of two elements in different summands is defined to be zero.) 
The natural action of $W_{0}$ on $\widehat{\mathscr{O}}$ (resp. $\widehat{\mathscr{O}}$ ) (see 7.3) corresponds under (a) and (b) to a $W_{0}$-action which permutes the summands $\widehat{\widehat{\mathscr{O}}}_{t}$ (resp. $\hat{\overline{\mathscr{O}}}_{\bar{t}}$ ) according to the transitive $W_{0}$-action on $\Sigma$ (resp. $\bar{\Sigma}$ ).

7.6. In the setup of 7.5, let $\mathscr{O}^{\text {an }}$ (resp. $\overline{\mathscr{O}}^{\text {an }}$ ) be the algebra of holomorphic functions $\mathscr{T} \times \mathbf{C}^{*} \rightarrow \mathbf{C}$ (resp. $\mathfrak{t} \oplus \mathbf{C} \rightarrow \mathbf{C}$ ), let $I_{(t)}^{\text {an }}$ (resp. $\bar{I}_{(t)}^{\text {an }}$ ) be its maximal ideal defined by $\left(t, v_{0}\right)$ (resp. $\left.\left(\bar{t}, r_{0}\right)\right)$, and let $\hat{\mathscr{O}}_{t}^{\text {an }}$ (resp. $\hat{\mathscr{O}}_{t}^{\text {an }}$ ) be the corresponding $I_{(t)}^{\text {an }}$-adic (resp. $\bar{I}_{(\bar{t})}^{\text {an }}$-adic) completion.

It is clear that we have natural isomorphisms

(a) $\hat{\mathscr{O}}_{t} \cong \hat{\mathscr{O}}_{t}^{\text {an }}, \widehat{\mathscr{O}}_{\bar{t}} \cong \widehat{\mathscr{O}}_{\bar{t}}^{\text {an }}$.

\section{FIRST REDUCTION THEOREM}

8.1. In this section, we preserve the setup of $\S 3$. We assume given a $W_{0}$-orbit $\Sigma$ in $\mathscr{T}$ and an element $v_{0} \in \mathbf{C}^{*}$. Let $\left\langle v_{0}\right\rangle$ be the subgroup of $\mathbf{C}^{*}$ generated by $v_{0}$, and let $\mathscr{T}\left\langle v_{0}\right\rangle$ be the subgroup $Y \otimes\left\langle v_{0}\right\rangle$ of $Y \otimes \mathbf{C}^{*}=\mathscr{T}$. Clearly, $\mathscr{T}\left\langle v_{0}\right\rangle$ is $W_{0}$-stable.

For any $t \in \mathscr{T}$, we define

$$
\begin{aligned}
R_{t} & =\left\{\alpha \in R \mid \begin{array}{ll}
\theta_{\alpha}(t) \in\left\langle v_{0}\right\rangle, & \text { if } \check{\alpha} \notin 2 Y \\
\theta_{\alpha}(t) \in \pm\left\langle v_{0}\right\rangle, & \text { if } \check{\alpha} \in 2 Y
\end{array}\right\}, \\
\check{R}_{t} & =\left\{\check{\alpha} \in \check{R} \mid \alpha \in R_{t}\right\}, \\
R_{t}^{+} & =R_{t} \cap R^{+}, \\
\Pi_{t} & =\text { set of all } \alpha \in R_{t}^{+} \text {which are not of the form } \alpha^{\prime}+\alpha^{\prime \prime} \text { with } \alpha^{\prime}, \alpha^{\prime \prime} \in R_{t}^{+}, \\
W_{0}^{t} & =\text { subgroup of } W_{0} \text { generated by the } s_{\alpha}\left(\alpha \in R_{t}\right), \\
\widetilde{W}_{0}^{t} & =\left\{w \in W_{0} \mid w(t)=t\right\}, \\
\Gamma_{t} & =\left\{w \in \widetilde{W}_{0}^{t} \mid w\left(R_{t}^{+}\right)=R_{t}^{+}\right\} .
\end{aligned}
$$

Note that $\left(X, Y, R_{t}, \check{R}_{t}, \Pi_{t}\right)$ is a root system. (We must check that $\alpha, \beta \in$ $R_{t} \Rightarrow s_{\beta}(\alpha) \in R_{t}$. We have

$$
\theta_{s_{\beta}(\alpha)}(t)=\theta_{\alpha}\left(s_{\beta}(t)\right)=\theta_{\alpha}\left(t \cdot h_{\check{\beta}}\left(\theta_{\beta}(t)\right)^{-1}\right)=\theta_{\alpha}(t) \theta_{\beta}(t)^{-\langle\alpha, \check{\beta}\rangle} .
$$

We have $\theta_{\beta}(t)^{-\langle\alpha, \ddot{\beta}\rangle} \in\left\langle v_{0}\right\rangle$ since $\beta \in R_{t}$. Hence, $\theta_{s_{\beta}(\alpha)}(t) \in \theta_{\alpha(t)} \cdot\left\langle v_{0}\right\rangle$ and our assertion follows.) Note also that $\Pi_{t} \not \subset \Pi$ in general. Clearly, $W_{0}^{t}$ is a normal subgroup of $\widetilde{W}_{0}^{t}$ with complement $\Gamma_{t}$.

Note that

(a) $R_{t}, \check{R}_{t}, \ldots, \Gamma_{t}$ depend only on the $\mathscr{T}\left\langle v_{0}\right\rangle$-coset of $t$, not on $t$ itself.

We define an equivalence relation on $\Sigma$ as follows. We say that $t, t^{\prime} \in \Sigma$ are equivalent if $t, t^{\prime}$ are in the same $\mathscr{T}\left\langle v_{0}\right\rangle$-coset and $t^{\prime}=w(t)$ for some $w \in W_{0}^{t}=W_{0}^{t^{\prime}}$. 
Let $\mathscr{P}$ be the set of equivalence classes. It is clear that if $t, t^{\prime} \in \Sigma$ are equivalent and $w \in W_{0}$, then $w(t), w\left(t^{\prime}\right)$ are equivalent. Hence, $W_{0}$ permutes (transitively) the sets in $\mathscr{P}$. For $c \in \mathscr{P}$, let

$$
W_{0}(c)=\left\{w \in W_{0} \mid w c=c\right\}
$$

be the isotropy group of $c$.

If $c \in \mathscr{P}$, we shall write $R_{c}, \check{R}_{c}, \check{R}_{c}^{+}, \Pi_{c}, W_{0}^{c}, \widetilde{W}_{0}^{c}, \Gamma_{c}$ instead of $R_{t}$, $\check{R}_{t}, \check{R}_{t}^{+}, \Pi_{t}, W_{0}^{t}, \widetilde{W}_{0}^{t}, \Gamma_{t}$ for $t \in c\left(\right.$ see 8.1(a)). Clearly, $W_{0}^{c} \subset W_{0}(c) \subset \widetilde{W}_{0}^{c}$. Let

$$
\Gamma(c)=W_{0}(c) \cap \Gamma_{c} .
$$

Then $W_{0}(c)$ is a semidirect product of $\Gamma(c)$ and the normal subgroup $W_{0}^{c}$.

Lemma 8.2. (a) If $Y_{0}$ is the subgroup of $Y$ generated by $\check{R}$, then we have $t \in \Sigma$, $w \in W_{0}^{t} \Rightarrow w(t) t^{-1} \in \operatorname{image}\left(Y_{0} \otimes\left\langle v_{0}\right\rangle \stackrel{\phi}{\rightarrow} Y \otimes \mathbf{C}^{*}\right) \quad(\phi$ is the natural map).

(b) If $\alpha \in R, c \in \mathscr{P}$, then $\alpha \in R_{c} \Leftrightarrow s_{\alpha}(c)=c$.

Proof. We prove (a). Since $Y_{0}$ is stable under $W_{0}$, we may assume that $w=s_{\beta}$ $\left(\beta \in R_{t}\right)$. We have $s_{\beta}(t) t^{-1}=h_{\check{\beta}}\left(\theta_{\beta}(t)^{-1}\right)=h_{\check{\beta}}\left(\nu v_{0}^{n}\right)$, where $n \in \mathbf{Z}$ and

$$
\nu= \begin{cases}1, & \text { if } \check{\beta} \notin 2 Y, \\ \pm 1, & \text { if } \check{\beta} \in 2 Y .\end{cases}
$$

If $\check{\beta} \in 2 Y$, we have $h_{\check{\beta}}(-1)=1$. Hence, in any case, $h_{\check{\beta}}(\nu)=1$ and $s_{\beta}(t) t^{-1}=h_{\check{\beta}}(-1)=\check{\beta} \otimes v_{0}^{n}$, as required.

We now prove (b). The implication $\Rightarrow$ is obvious. We prove the converse. Assume that $s_{\alpha}(c)=c$ and choose $t \in c$. Then $t, s_{\alpha}(t)$ are in the same $W_{0}^{t}$ orbit, i.e., $s_{\alpha}(t)=w(t)$ for some $w \in W_{0}^{t}$. Using (a), we have $s_{\alpha}(t)^{-1}=$ $w(t) \cdot t^{-1} \in$ image $(\phi)$. We set $u_{\alpha}=\theta_{\alpha}(t)^{-1} \in \mathbf{C}^{*}$. Then $\check{\alpha} \otimes u_{\alpha} \in$ image $\phi$. In particular, $\check{\alpha} \otimes u_{\alpha} \in Y \otimes\left\langle v_{0}\right\rangle$. If $\check{\alpha} \notin 2 Y$, then $\check{\alpha}$ is not divisible in $Y$ and it follows that $u_{\alpha} \in\left\langle v_{0}\right\rangle$, i.e., $\alpha \in R_{t}$. Assume now that $\check{\alpha} \in 2 Y$. Using Lemma 1.7 , we can assume that our root system is primitive. By properties of root systems of type $\mathrm{C}$, we see that we can number the roots in $\Pi$ as $\alpha_{1}, \ldots, \alpha_{n}$ such that $\check{\alpha}=\check{\alpha}_{1}+c_{2} \check{\alpha}_{2}+c_{3} \check{\alpha}_{3}+\cdots+c_{n} \check{\alpha}_{n}\left(c_{2}, \ldots, c_{n}\right.$ are integers $\left.\geq 0\right)$, where $\check{\alpha}_{1} \in 2 Y$ and $\frac{1}{2} \check{\alpha}_{1}, \check{\alpha}_{2}, \check{\alpha}_{3}, \ldots, \check{\alpha}_{n}$ form a basis of $Y$. From $\check{\alpha} \otimes u_{\alpha} \in$ image $\phi$, we have (writing now the operation in $Y \otimes \mathbf{C}^{*}$ as addition),

$$
\check{\alpha}_{1} \otimes u_{\alpha}+\sum_{i=2}^{n} c_{i} \check{\alpha}_{i} \otimes u_{\alpha}=\sum_{i=1}^{n} \check{\alpha}_{i} \otimes v_{0}^{d_{i}} \quad\left(d_{i} \in \mathbf{Z}\right) .
$$

Hence,

$$
\frac{\check{\alpha}_{1}}{2} \otimes u_{\alpha}^{2}+\sum_{i=2}^{n} \check{\alpha}_{i} \otimes u_{\alpha}^{c_{i}}=\frac{\check{\alpha}_{1}}{2} \otimes v_{0}^{2 d_{1}}+\sum_{i=2}^{n} \check{\alpha}_{i} \otimes v_{0}^{d_{i}} .
$$

Since $\check{\alpha}_{1} / 2, \check{\alpha}_{2}, \ldots, \check{\alpha}_{n}$ form a basis of $Y$, it follows that $u_{\alpha}^{2}=v_{0}^{2 d_{1}}$, so that $u_{\alpha}= \pm v_{0}^{d_{1}}$ and $\alpha \in R_{t}$. The lemma is proved. 
8.3. For $c \in \mathscr{P}$, we denote by $H_{c}$ the Hecke algebra defined in terms of the root system $\left(X, Y, R_{c}, \breve{R}_{c}, \Pi_{c}\right)$ and the parameter set (see 3.1(c)),

$$
\begin{aligned}
& \lambda_{c}(\alpha)=\lambda(\alpha) \quad\left(\alpha \in \Pi_{c}\right), \\
& \lambda_{c}^{*}(\alpha)=\lambda^{*}(\alpha) \quad\left(\alpha \in \Pi_{c}, \check{\alpha} \in 2 Y\right)
\end{aligned}
$$

$\left(\lambda(\alpha), \lambda^{*}(\alpha)\right.$ as in 3.13) in the same way that $H$ was defined in terms of $(X, Y, R, \check{R}, \Pi)$ and the parameter set $\lambda, \lambda^{*}$.

Since $\Gamma(c)$ acts on $\left(X, Y, R_{c}, \check{R}_{c}, \Pi_{c}\right)$ compatibly with the parameter set (since $\left.\Gamma(c) \subset W_{0}\right)$, it also acts naturally on the algebra $H_{c}$ : if $T_{w, c} \theta_{x}\left(w \in W_{0}^{c}\right.$, $x \in X)$ are the basis elements of $H_{c}$ analogous to the basis elements $T_{w} \theta_{x}$ $\left(w \in W_{0}, x \in X\right)$ of $H$ and $\gamma \in \Gamma(c)$, we have $\gamma\left(T_{w, c} \theta_{x}\right)=T_{\gamma w \gamma^{-1}, c} \theta_{\gamma(x)}$.

8.4. Consider the maximal ideal $J_{\left(\Sigma, v_{0}\right)}$ of $\mathscr{Z}$ (see 7.2(a)). Similarly, for $c \in \mathscr{P}$, we consider the maximal ideal

$$
J_{\left(c, v_{0}\right)}=\left\{f \in \mathscr{Z}_{c} \mid f\left(t, v_{0}\right)=0, \forall t \in c\right\}
$$

of $\mathscr{Z}_{c}=\mathscr{O}^{W_{0}^{c}}=$ center $\left(H_{c}\right)$. (Note that $c$ is a $W_{0}^{c}$-orbit in $\mathscr{T}$.) In 7.3, we introduced the $J_{\left(\Sigma, v_{0}\right)}$-adic completions $\widehat{\mathscr{Z}}, \widehat{\mathscr{O}}, \widehat{H}$ of the $\mathscr{Z}$-algebras $\mathscr{Z}, \mathscr{O}$, $H$. Similarly, let $\widehat{\mathscr{Z}}_{c}, \widehat{\mathscr{O}}_{c}, \widehat{H}_{c}$ be the $J_{\left(c, v_{0}\right)}$-adic completions of the $\mathscr{Z}_{c}$-algebras $\mathscr{Z}_{c}, \mathscr{O}, H_{c}$.

We shall also need $\widehat{K}, \widehat{H}_{F}$ of 7.3 and 7.4.

The action of $\Gamma(c)$ on $H_{c}$ extends continuously to an action of $\Gamma(c)$ on $\widehat{H}_{c}$, since $\Gamma(c)$ leaves stable the maximal ideal $J_{\left(c, v_{0}\right)}$ of $\mathscr{Z}_{c}$. (Recall that $\Gamma(c) \subset W_{0}(c)$.)

8.5. If $A$ is an associative ring with 1 , denote by $A_{n}$ the ring of all $n \times n$ matrices with entries in $A$. If a finite group $\Gamma$ acts on $A$ by ring automorphisms, we can define formally a new ring $A[\Gamma]=\bigoplus_{\gamma \in \Gamma} A \cdot \gamma$ with multiplication $(a \gamma)\left(a^{\prime} \gamma^{\prime}\right)=\left(a \gamma\left(a^{\prime}\right)\right) \cdot\left(\gamma \gamma^{\prime}\right)$. (The group algebra of $\Gamma$ over a field is a special case of this.) In particular, the action of $\Gamma(c)$ on $\widehat{H}_{c}$ gives rise to a ring $\widehat{H}_{c}[\Gamma(c)]$. This is not, in general, a $\widehat{\mathscr{Z}}_{c}$-algebra since $\Gamma(c)$ may act nontrivially on $\widehat{\mathscr{Z}}_{c}$. It is only a $\widehat{\mathscr{Z}}_{c}^{\Gamma(c)}$-algebra.

We have $\widehat{\mathscr{Z}}_{c}^{\Gamma(c)}=\left(\hat{\mathscr{O}}_{c}^{W_{0}^{c}}\right)^{\Gamma(c)}=\widehat{\mathscr{O}}_{c}^{W_{0}(c)}$. Thus, $\widehat{H}_{c}[\Gamma(c)]$ is a $\hat{\mathscr{O}}_{c}^{W_{0}(c)}$-algebra. The identity map $\mathscr{O} \rightarrow \mathscr{O}$ extends continuously to a ring homomorphism $\widehat{\mathscr{O}} \rightarrow$ $\hat{\mathscr{O}}_{c}$ (since $\left.J_{\left(\Sigma, v_{0}\right)} \subset J_{\left(c, v_{0}\right)}\right)$. This restricts to a ring homomorphism $i: \hat{\mathscr{O}}^{W_{0}} \rightarrow$ $\hat{\mathscr{O}}_{c}^{W_{0}(c)}$ (since $W_{0}(c) \subset W_{0}$ ). It is clear that $i$ is an isomorphism. (By 7.5(a) and its analogue for $\widehat{\mathscr{O}}_{c}$, both $\hat{\mathscr{O}}^{W_{0}}$ and $\hat{\mathscr{O}}_{c}^{W_{0}(c)}$ are isomorphic to the ring of invariants on $\hat{\mathscr{O}}_{t}$ (for some $t \in c$ ) with respect to the stabilizer of $t$ in $W_{0}$ (or in $\left.W_{0}(c)\right)$. Via $i$, we can regard $\widehat{H}_{c}[\Gamma(c)]$ also as a $\hat{\mathscr{O}}^{W_{0}}$-algebra. 
We can now state

Theorem 8.6. If $c \in \mathscr{P}$, there exists an isomorphism of $\hat{\mathscr{O}}^{W_{0}}$-algebras $\hat{H} \cong$ $\widehat{H}_{c}[\Gamma(c)]_{n}$, where $n=\# \mathscr{P}$.

The proof will occupy most of this section.

8.7. Recall the decomposition $\widehat{\mathscr{O}}=\bigoplus_{t \in \Sigma} \widehat{\mathscr{O}}_{t}$ (in 7.5(a)). For any $t \in \Sigma$, we denote by $1_{t}$ the unit element of $\widehat{\mathscr{O}}_{t}$. We also regard $1_{t}$ as an element of $\widehat{\mathscr{O}}$. Then the unit element 1 of $\widehat{\mathscr{O}}$ and of $\hat{H}$ satisfies $1=\sum_{t \in \Sigma} 1_{t}, 1_{t} \cdot 1_{t^{\prime}}=\delta_{t, t^{\prime}} \cdot 1_{t}$ $\left(t, t^{\prime} \in \Sigma\right)$ and $w\left(1_{t}\right)=1_{w(t)}\left(w \in W_{0}, t \in \Sigma\right)$.

Let $c \in \mathscr{P}$. We define

(a) $1_{c}=\sum_{t \in c} 1_{t} \in \hat{\mathscr{O}} \subset \widehat{H}$.

We may identify $\hat{\mathscr{O}}_{c}$ (see 8.5) with the subring $1_{c} \widehat{\mathscr{O}}=\widehat{\mathscr{O}} 1_{c}=\bigoplus_{t \in c} \hat{\mathscr{O}}_{t}$ in the obvious way.

It is clear that

(b) $1=\sum_{c \in \mathscr{P}} 1_{c}, 1_{c} \cdot 1_{c^{\prime}}=\delta_{c, c^{\prime}} \cdot 1_{c}\left(c, c^{\prime} \in \mathscr{P}\right), w\left(1_{c}\right)=1_{w(c)}\left(w \in W_{0}\right.$, $c \in \mathscr{P})$.

For $c, c^{\prime} \in \mathscr{P}$, we define

(c) $\widehat{H}_{c^{\prime}}=1_{c} \widehat{H} 1_{c^{\prime}} \subset \widehat{H}$.

From (b), we see that

(d) $\hat{\mathscr{O}}=\bigoplus_{c \in \mathscr{P}} \hat{\mathscr{O}}_{c}, \hat{\mathscr{O}}_{c} \widehat{\mathscr{O}}_{c} \subset \widehat{\mathscr{O}}_{c}, \widehat{\mathscr{O}}_{c} \widehat{\mathscr{O}}_{c^{\prime}}=0$ if $c \neq c^{\prime}$.

(e) $\hat{H}=\bigoplus_{c, c^{\prime} \in \mathscr{P}}{ }_{c} \widehat{H}_{c^{\prime}},{ }_{c} \widehat{H}_{c^{\prime}} \cdot{ }_{c^{\prime}} \widehat{H}_{c^{\prime \prime}} \subset{ }_{c} \widehat{H}_{c^{\prime \prime}},{ }_{c} \widehat{H}_{c^{\prime}} \cdot{ }_{c_{1}^{\prime}} \widehat{H}_{c^{\prime \prime}}=0$ if $c^{\prime} \neq c_{1}^{\prime}$.

8.8. Let $\alpha \in \Pi, c \in \mathscr{P}$. We define an element $T_{s_{\alpha}}^{c} \in \widehat{H}_{F}$ by

(a)

$$
T_{s_{\alpha}}^{c}= \begin{cases}1_{c} T_{s_{\alpha}}=T_{s_{\alpha}} 1_{s_{\alpha}}(c), & \text { if } \left.\alpha \in R_{c} \text { (i.e., if } s_{\alpha}(c)=c\right), \\ 1_{c} \tau^{\alpha}=\tau^{\alpha} 1_{s_{\alpha}}(c), & \text { if } \left.\alpha \notin R_{c} \text { (i.e., if } s_{\alpha}(c) \neq c\right)\end{cases}
$$

(we use $s_{\alpha}\left(1_{c}\right)=1_{s_{\alpha}(c)}$ and Lemma 8.2(b)).

Lemma 8.9. (a) If $\alpha \in \Pi, \alpha \notin R_{c}$, then the rational function $\mathscr{G}(\alpha)$ on $\mathscr{T}$ is regular and nonzero at all points of $c \cup s_{\alpha}(c)$.

(b) We have $T_{s_{\alpha}}^{c} \in \widehat{c}_{s_{\alpha}(c)}$ for all $\alpha \in \Pi$.

Proof. Let $\alpha \in \Pi, \alpha \notin R_{c}$, and let $t \in c$. We show that the numerator and denominator of $\mathscr{G}(\alpha)$ (see 3.8) do not vanish at $t$ and $s_{\alpha}(t)$, i.e.,

$$
\left\{\begin{array}{l}
\theta_{\alpha}(t)^{ \pm 1} v_{0}^{2 \lambda(\alpha)}-1 \neq 0, \theta_{\alpha}(t)^{ \pm 1}-1 \neq 0, \quad \text { if } \check{\alpha} \notin 2 Y, \\
\theta_{\alpha}(t)^{ \pm 1} v_{0}^{\lambda(\alpha)+\lambda^{*}(\alpha)}-1 \neq 0, \theta_{\alpha}(t)^{ \pm 1} v_{0}^{\lambda(\alpha)-\lambda^{*}(\alpha)}+1 \neq 0, \theta_{\alpha}^{ \pm 2}(t)-1 \neq 0, \\
\text { if } \check{\alpha} \in 2 Y .
\end{array}\right.
$$

(We have $\theta_{\alpha}\left(s_{\alpha}(t)\right)=\theta_{\alpha}(t)^{-1}$.) But this follows from $\alpha \notin R_{c}$. This proves (a). Now (b) is clear if $\alpha \in R_{c}$. Assume now that $\alpha \notin R_{c}$. We have $T_{s_{\alpha}}^{c}=$ $1_{c} \tau^{a} 1_{s_{\alpha}(c)}=1_{c}\left(\tau^{\alpha}+1\right) 1_{s_{\alpha}(c)}\left(\right.$ since $\left.1_{c} 1_{s_{\alpha}(c)}=0\right)$. Hence, using 5.1(a), $T_{s_{\alpha}}^{c}=$ 
$1_{c}\left(T_{s_{\alpha}}+1\right) \mathscr{G}(\alpha)^{-1} 1_{s_{\alpha}(c)}$. From (a), we see that $\mathscr{G}(\alpha)^{-1} 1_{s_{\alpha}(c)} \in \widehat{\mathscr{O}}_{s_{\alpha}(c)}$ and (b) follows.

8.10. Given $c \in \mathscr{P}$ and $w \in W_{0}$, we define

(a) $T_{w}^{c}=T_{s_{\alpha_{1}}}^{c} T_{s_{\alpha_{1}}}^{s_{\alpha_{1}}(c)} \cdots T_{s_{\alpha_{p}}}^{s_{\alpha_{p}} \cdots s_{\alpha_{2}} s_{\alpha_{1}}(c)}$,

where $\alpha_{1}, \alpha_{2}, \ldots, \alpha_{p}$ is any sequence in $\Pi$ such that $w=s_{\alpha_{1}} s_{\alpha_{2}} \cdots s_{\alpha_{p}}, p=$ $l(w)$.

From Lemma 8.9 and $8.7(\mathrm{e})$, we see that

(b) $T_{w}^{c} \in{ }_{c} \widehat{H}_{w^{-1}(c)}$.

Proposition 8.11. The element $T_{w}^{c}$ is well defined (it is independent of the choice of $\left.\alpha_{1}, \alpha_{2}, \ldots, \alpha_{p}\right)$.

Proof. For any $\alpha \in \Pi$, we define

$$
\widetilde{T}_{s_{\alpha}}=\sum_{c \in \mathscr{P}} T_{s_{\alpha}}^{c} \in \widehat{H}
$$

We first show that for any $\alpha \neq \beta$ in $\Pi$ such that $s_{\alpha} s_{\beta}$ has order $m$, we have

(a) $\tilde{T}_{s_{\alpha}} \widetilde{T}_{s_{\beta}} \widetilde{T}_{s_{\alpha}} \cdots=\widetilde{T}_{s_{\beta}} \widetilde{T}_{s_{\alpha}} \widetilde{T}_{s_{\beta}} \ldots$

(both products have $m$ factors).

This is equivalent to the identity

$$
\sum_{c \in \mathscr{P}}\left(T_{s_{\alpha}}^{c} T_{s_{\beta}}^{s_{\alpha}(c)} T_{s_{\alpha}}^{s_{\beta} s_{\alpha}(c)} \cdots\right)=\sum_{c \in \mathscr{P}}\left(T_{s_{\beta}}^{c} T_{s_{\alpha}}^{s_{\beta}(c)} T_{s_{\beta}}^{s_{\alpha} s_{\beta}(c)} \ldots\right),
$$

where all products have $m$ factors. (We have used Lemma 8.9 and $8.7(\mathrm{e})$ ). Therefore, to prove (a) it is enough to show that for any fixed $c \in \mathscr{P}$, we have

$$
T_{s_{\alpha}}^{c} T_{s_{\beta}}^{s_{\alpha}(c)} T_{s_{\alpha}}^{s_{\beta} s_{\alpha}(c)} \ldots=T_{s_{\beta}}^{c} T_{s_{\alpha}}^{s_{\beta}(c)} T_{s_{\beta}}^{s_{\alpha} s_{\beta}(c)} \ldots
$$

Using the definition $8.7(\mathrm{a})$, we see that this is equivalent to

$$
1_{c} \xi_{1} \xi_{2} \cdots \xi_{m}=1_{c} \eta_{1} \eta_{2} \cdots \eta_{m}
$$

where $\xi_{i}, \eta_{i}$ are as in Proposition 6.2. (We have used 8.7(b).) This is a consequence of Proposition 6.2. Thus, (a) is proved. By a well-known property of the braid group of $W_{0}$, we see from (a) that we can define for $w \in W_{0}$

$$
\widetilde{T}_{w}=\widetilde{T}_{s_{\alpha_{1}}} \widetilde{T}_{s_{\alpha_{2}}} \cdots \widetilde{T}_{s_{\alpha_{p}}} \in \widehat{H}
$$

where $\alpha_{1}, \alpha_{2}, \ldots, \alpha_{p}$ is any sequence in $\Pi$ such that $w=s_{\alpha_{1}} s_{\alpha_{2}} \cdots s_{\alpha_{p}}, l(w)=$ $p$ (and this is independent of the choice). Then $T_{w}^{c}$ as defined in 8.10(a) is the projection of $\widetilde{T}_{w}$ onto the ${ }_{c} \widehat{H}_{w^{-1}(c)}$-summand in the decomposition $\widehat{H}=$ $\bigoplus_{c} \widehat{H}_{c^{\prime}}$. Hence, it is intrinsically defined. This completes the proof of the proposition.

Lemma 8.12. Let $c \in \mathscr{P}$, and let $\alpha \in \Pi_{c}$ be such that $l\left(s_{\alpha}\right)>1$ (length in $\left.W_{0}\right)$. Then

(a) there exists $\beta \in \Pi$ such that $l\left(s_{\beta} s_{\alpha} s_{\beta}\right)=l\left(s_{\alpha}\right)-2$.

(b) If $\beta$ is as in (a), then $\beta \notin R_{c}^{+}$. 
Proof. (Compare [2].) Being a reflection, $s_{\alpha}$ has odd length. Hence, $l\left(s_{\alpha}\right) \geq 3$. Let $\beta \in \Pi$ be such that $l\left(s_{\beta} s_{\alpha}\right)=l\left(s_{\alpha}\right)-1$. Then $l\left(s_{\alpha} s_{\beta}\right)=l\left(s_{\beta} s_{\alpha}\right)=l\left(s_{\alpha}\right)-1$. We can find a sequence $\alpha_{1}, \ldots, \alpha_{p}$ in $\Pi$ such that $s_{\alpha}=s_{\beta} s_{\alpha_{1}} s_{\alpha_{2}} \cdots s_{\alpha_{p}}, l\left(s_{\alpha}\right)=$ $p+1$. Since $l\left(s_{\alpha} s_{\beta}\right)=l\left(s_{\alpha}\right)-1$, we see from the exchange condition that either there exists $m \geq 1$ with

$$
s_{\alpha_{m}} s_{\alpha_{m+1}} \cdots s_{\alpha_{p}}=s_{\alpha_{m+1}} s_{\alpha_{m+2}} \cdots s_{\alpha_{p}} s_{\beta}
$$

or

$$
s_{\beta} s_{\alpha_{1}} s_{\alpha_{2}} \cdots s_{\alpha_{p}}=s_{\alpha_{1}} s_{\alpha_{2}} \cdots s_{\alpha_{p}} s_{\beta} .
$$

In the first case, we have $s_{\alpha}=s_{\beta} s_{\alpha_{1}} \cdots s_{\alpha_{m-1}} s_{\alpha_{m+1}} s_{\alpha_{m+2}} \cdots s_{\alpha_{p}} s_{\beta}$ and (a) follows. In the second case, we have $s_{\alpha}=s_{\alpha_{1}} s_{\alpha_{2}} \cdots s_{\alpha_{p}} s_{\beta}=s_{\beta} s_{\alpha} s_{\beta}=s_{s_{\beta}(\alpha)}$. Hence, $\alpha, s_{\beta}(\alpha)$ are proportional. Hence, $s_{\beta}(\alpha)= \pm \alpha$. We cannot have $s_{\beta}(\alpha)=-\alpha$ : the only positive root taken by $s_{\beta}$ to a negative one is $\beta$ (recall that $\alpha \notin \Pi$, so $\alpha \neq \beta)$. Hence, $s_{\beta}(\alpha)=\alpha$, so $\langle\alpha, \check{\beta}\rangle=0$. But then $\langle\beta, \check{\alpha}\rangle=0$. Hence, $s_{\alpha}(\beta)=\beta \in R^{+}$, so $l\left(s_{\alpha} s_{\beta}\right)=l\left(s_{\alpha}\right)+1$, a contradiction. Thus, (a) is proved. We now prove (b). Assume that $\beta \in R_{c}^{+}$. From $l\left(s_{\alpha} s_{\beta}\right)=l\left(s_{\alpha}\right)-1$, it follows that $s_{\alpha}(\beta) \in R^{-}$. Thus, $s_{\alpha}$ carries two nonproportional roots in $R_{c}^{+}$(namely, $\alpha$ and $\beta$ ) to negative ones. This contradicts the fact that $\alpha \in \Pi_{c}$. The lemma is proved.

Lemma 8.13. Let $c \in \mathscr{P}, \gamma \in \Gamma(c)$, and let $\alpha_{1}, \alpha_{2}, \ldots, \alpha_{p} \in \Pi$ be such that $\gamma=s_{\alpha_{1}} s_{\alpha_{2}} \cdots s_{\alpha_{p}}, l(\gamma)=p$. Then

(a) $c \neq s_{\alpha_{1}}(c) \neq s_{\alpha_{2}} s_{\alpha_{1}}(c) \neq \cdots \neq s_{\alpha_{p}} \cdots s_{\alpha_{2}} s_{\alpha_{1}}(c)$ (i.e., $\alpha_{1} \notin R_{c}, s_{\alpha_{1}}\left(\alpha_{2}\right) \notin$ $\left.R_{c}, \ldots, s_{\alpha_{1}} s_{\alpha_{2}} \ldots s_{\alpha_{p-1}}\left(\alpha_{p}\right) \notin R_{c}\right)$.

(b) $T_{\gamma}^{c}=1_{c} \tau^{\alpha_{1}} \tau^{\alpha_{2}} \cdots \tau^{\alpha_{p}} 1_{c}$.

(c) $T_{\gamma}^{c} T_{w}^{c}=T_{\gamma w}^{c}, T_{w}^{c} T_{\gamma}^{c}=T_{w \gamma}^{c}$, for all $w \in W_{0}^{c}$.

(d) $T_{\gamma}^{c} f 1_{c}=1_{c} \gamma(f) T_{\gamma}^{c}$, for all $f \in \widehat{\mathscr{O}}$.

Proof. We first note

(e) if $y \in W_{0}, \beta \in \Pi, s_{\beta}(c) \neq c$, then $\tau^{\beta} T_{y}^{c}=T_{s_{\beta} y}^{s_{\beta}(c)}$.

Indeed, if $l\left(s_{\beta} y\right)=l(y)+1$, this follows from definitions (8.8 and 8.10). If $l\left(s_{\beta} y\right)=l(y)-1$, the same definitions show that $T_{y}^{c}=\tau^{\beta} T_{s_{\beta} y}^{s_{\beta}(c)}$. Multiplying on both sides by $\tau^{\beta}$ and using $\tau^{\beta} \tau^{\beta}=1$, we again find (e).

Assume now that we have $\delta=s_{\alpha_{1}} s_{\alpha_{2}} \cdots s_{\alpha_{i-1}}\left(\alpha_{i}\right) \in R_{c}$ for some $i \in[1, p]$. Since $l\left(s_{\alpha_{1}} \cdots s_{\alpha_{i}}\right)=i$, we have $\delta \in R^{+}$. Hence, $\delta \in R_{c}^{+}$. We have $\alpha_{i}=$ $s_{\alpha_{i-1}} \cdots s_{\alpha_{1}}(\beta)$. Now $s_{\alpha_{p}} \cdots s_{\alpha_{i+1}}\left(\alpha_{i}\right) \in R^{+}$since $l\left(s_{\alpha_{p}} \cdots s_{\alpha_{i+1}} \alpha_{i}\right)=p-i+1$. Hence, $\gamma^{-1}(\delta)=s_{\alpha_{p}} \cdots s_{\alpha_{2}} s_{\alpha_{1}}(\delta)=s_{\alpha_{p}} \cdots s_{\alpha_{i+1}} s_{\alpha_{i}}\left(\alpha_{i}\right)=-s_{\alpha_{p}} \cdots s_{\alpha_{i+1}}\left(\alpha_{i}\right) \in R^{-}$. By assumption, $\gamma \in \Gamma_{c}$. Hence, $\gamma\left(R_{c}^{+}\right)=R_{c}^{+}$. This contradicts $\gamma^{-1}(\delta) \in R^{-}$. This contradiction proves (a). Now (b) follows from (a) using the definitions 8.8 and 8.10. Using (e) repeatedly, we see that (c) follows from (b). Using the 
identity $f \tau^{\alpha}=\tau^{\alpha} s_{\alpha}(f)$ in $\widehat{H}_{F}(f \in \widehat{\mathscr{O}}, \alpha \in \Pi)$, we see that (d) follows from (b). The lemma is proved.

Lemma 8.14. Let $c \in \mathscr{P}, \alpha \in \Pi_{c}$.

(a) We have $\left(T_{s_{\alpha}}^{c}+1\right)\left(T_{s_{\alpha}}^{c}-v^{2 \lambda(\alpha)}\right)=0$ in $\widehat{H}(\lambda(a)$ as in 3.13).

(b) If $w \in W_{0}^{c}$ is such that $w^{-1}(\alpha) \in R_{c}^{+}$, then $T_{s_{\alpha}}^{c} T_{w}^{c}=T_{s_{\alpha} w}^{c}$.

(c) If $f \in \widehat{\mathscr{O}}$, then $f T_{s_{\alpha}}^{c}=T_{s_{\alpha}}^{c} s_{\alpha}(f)+1_{c}\left(f-s_{\alpha}(f)\right)(\mathscr{G}(\alpha)-1)$ in $\hat{H}$.

Proof. We argue by induction of $l\left(s_{\alpha}\right)$. If $l\left(s_{\alpha}\right)=1$, we have $T_{s_{\alpha}}^{c}={ }_{c} T_{s_{\alpha}}$. Hence, (a) and (c) follow from 3.2(b) and 3.12(d); (b) follows from 8.8(a) and 8.10 (a). Now assume that $l\left(s_{\alpha}\right)>1$. Choose $\beta \in \Pi$ so that $\alpha^{\prime}=s_{\beta}(\alpha)$ satisfies $l\left(s_{\alpha^{\prime}}\right)=l\left(s_{\alpha}\right)-2($ see Lemma 8.12(a)).

Set $c^{\prime}=s_{\beta}(c)$. By Lemma 8.12(b), we have $\beta \notin R_{c}^{+}$. Hence, $s_{\beta}\left(R_{c}^{+}\right) \subset R^{+}$. (Recall that the only positive root taken by $s_{\beta}$ to a negative one is $\beta$.) Hence, $s_{\beta}\left(R_{c}^{+}\right)=R_{c^{\prime}}^{+}$and $s_{\beta}\left(\Pi_{c}\right)=\Pi_{c^{\prime}}$. In particular, $\alpha^{\prime} \in \Pi_{c^{\prime}}$. We may assume that the lemma is true for $\left(\alpha^{\prime}, c^{\prime}\right)$ instead of $(\alpha, c)$, since $l\left(s_{\alpha^{\prime}}\right)<l\left(s_{\alpha}\right)$.

We have

(d) $T_{s_{\alpha}}^{c}=\tau^{\beta} T_{s_{\alpha^{\prime}}}^{c^{\prime}} \tau^{\beta}$

(using $c \neq s_{\beta}(c)=c^{\prime}$ which follows from Lemma 8.12(b) and using 8.10(a)).

Now (a) and (c) follow from the corresponding identities for $T_{s_{\alpha^{\prime}}}^{c^{\prime}}$ using (d) and $\tau^{\beta} \tau^{\beta}=1$. We now prove (b). Let $w^{\prime}=s_{\beta} w s_{\beta} \in W_{0}^{c^{\prime}}$.

We have

$$
w^{\prime^{-1}}\left(\alpha^{\prime}\right)=s_{\beta} w^{-1} s_{\beta}\left(\alpha^{\prime}\right)=s_{\beta}\left(w^{-1}(\alpha)\right) \subset s_{\beta}\left(R_{c}^{+}\right)=R_{c^{\prime}}^{+} .
$$

Hence, $T_{s_{\alpha^{\prime}}}^{c^{\prime}} T_{w^{\prime}}^{c^{\prime}}=T_{s_{\alpha^{\prime}} w^{\prime}}^{c^{\prime}}$, using the induction hypothesis.

From the definition 8.10(a), we see, using $c \neq s_{\beta}(c)=c^{\prime}$ (just as in 8.13(e)), that

$$
T_{w}^{c}=\tau^{\beta} T_{w^{\prime}}^{c^{\prime}} \tau^{\beta}
$$

We now compute

$$
T_{s_{\alpha}}^{c} T_{w}^{c}=\left(\tau^{\beta} T_{s_{\alpha^{\prime}}}^{c^{\prime}} \tau^{\beta}\right)\left(\tau^{\beta} T_{w^{\prime}}^{c^{\prime}} \tau^{\beta}\right)=\tau^{\beta} T_{s_{\alpha^{\prime}}}^{c^{\prime}} T_{w^{\prime}}^{c^{\prime}} \tau^{\beta}=\tau^{\beta} T_{s_{\alpha^{\prime}} w^{\prime}}^{c^{\prime}} \tau^{\beta}=T_{s_{\alpha} w}^{c} .
$$

The lemma is proved.

Lemma 8.15. Let $c, c^{\prime} \in \mathscr{P}$. Then ${ }_{c} \widehat{H}_{c^{\prime}}=\bigoplus_{w \in W_{0} ; w(c)=c^{\prime}}\left(\widehat{\mathscr{O}}_{c} \cdot T_{w}^{c}\right)$.

Proof. We shall prove by induction on $l(w)$ that

(a) $1_{c} T_{w} 1_{c^{\prime}} \subset{ }_{c} \widetilde{H}_{c^{\prime}}\left(\forall w \in W_{0}\right)$, where

(b) ${ }_{c} \widetilde{H}_{c^{\prime}}=\sum_{w^{\prime} \in W_{0} ; w^{\prime}(c)=c^{\prime}} \widehat{\mathscr{O}}_{c} T_{w^{\prime}}^{c}$.

When $w=1$, (a) is trivial. Hence, we may assume that $w=s_{\alpha} w_{1}$, where $\alpha \in \Pi, l(w)=l\left(w_{1}\right)+1$ and that (a) is known for $w_{1}$ instead of $w$. Assume first that $\alpha \in R_{c}$. Then

$$
1_{c} T_{w} 1_{c^{\prime}}=1_{c} T_{s_{\alpha}} T_{w_{1}} 1_{c^{\prime}}=T_{s_{\alpha}} 1_{c} T_{w_{1}} 1_{c^{\prime}} \in T_{s_{\alpha} c} \widetilde{H}_{c^{\prime}}
$$


We have $T_{s_{\alpha}} \hat{\mathscr{O}} \subset \hat{\mathscr{O}} T_{s_{\alpha}}+\hat{\mathscr{O}}$. Hence, $T_{s_{\alpha}} \hat{\mathscr{O}}_{c} \subset \hat{\mathscr{O}}_{c} T_{s_{\alpha}}+\hat{\mathscr{O}}_{c}$ and $1_{c} T_{w} 1_{c^{\prime}} \in$ $\sum_{w^{\prime}} \widehat{\mathscr{O}}_{c} T_{s_{\alpha}} T_{w^{\prime}}^{c}+\sum_{w^{\prime}} \widehat{\mathscr{O}}_{c} T_{w^{\prime}}^{c} \quad\left(w^{\prime}(c)=c^{\prime}\right.$ in the summation). If $l\left(s_{\alpha} w^{\prime}\right)=$ $l\left(w^{\prime}\right)+1$, we have $T_{s_{\alpha}} T_{w^{\prime}}^{c}=T_{s_{\alpha} w^{\prime}}^{c}$. If $l\left(s_{\alpha} w^{\prime}\right)=l\left(w^{\prime}\right)-1$, we have $T_{s_{\alpha}} T_{w^{\prime}}^{c}=$ $T_{s_{\alpha}}^{c} T_{s_{\alpha}}^{c} T_{s_{\alpha} w^{\prime}}^{c}=\left(v^{2 \lambda(\alpha)}-1\right) T_{w^{\prime}}^{c}+v^{2 \lambda(\alpha)} T_{s_{\alpha} w^{\prime}}^{c}$ (see Lemma 8.14), and we see that $1_{c} T_{w} 1_{c^{\prime}} \subset{ }_{c} \widetilde{H}_{c^{\prime}}$.

Assume next that $\alpha \notin R_{c}$. Then

$$
\begin{aligned}
1_{c} T_{w} 1_{c^{\prime}}= & 1_{c} T_{s_{\alpha}} T_{w_{1}} 1_{c^{\prime}}=1_{c}\left(\tau^{\alpha} \mathscr{G}(\alpha)+(\mathscr{G}(\alpha)-1)\right) T_{w_{1}} 1_{c^{\prime}} \\
= & \tau^{\alpha} \mathscr{G}(\alpha) 1_{s_{\alpha}}(c) T_{w_{1}} 1_{c^{\prime}}+(\mathscr{G}(\alpha)-1) 1_{c} T_{w_{1}} 1_{c^{\prime}} \\
& \in \tau^{\alpha} \mathscr{G}(\alpha) s_{\alpha}(c) \widetilde{H}_{c^{\prime}}+(\mathscr{G}(\alpha)-1) \widetilde{H}_{c^{\prime}}
\end{aligned}
$$

Now $\mathscr{G}(\alpha)$ is regular at all $t \in c \cup s_{\alpha}(c)$ (see Lemma 8.9(a)). Hence, $\mathscr{G}(\alpha)$ can be absorbed in ${ }_{s_{\alpha}(c)} \widetilde{H}_{c^{\prime}}$ and $\widetilde{H}_{c, c^{\prime}}$. Thus, $1_{c} T_{w} 1_{c^{\prime}} \in \tau_{s_{\alpha}(c)}^{\alpha} \widetilde{H}_{c^{\prime}}+{ }_{c} \widetilde{H}_{c^{\prime}}$. It remains to show that $\tau^{\alpha}{ }_{s_{\alpha}(c)} \widetilde{H}_{c^{\prime}} \subset_{c} \widetilde{H}_{c^{\prime}}$. This follows from the equality 8.13(e). Thus, (a) is proved. We now show that the sum defining ${ }_{c} \widetilde{H}_{c^{\prime}}$ in (b) is direct. It is enough to show

(c) a relation $\sum_{w^{\prime} \in W_{0}} f_{w^{\prime}} T_{w^{\prime}}^{c}=0 \quad\left(f_{w^{\prime}} \in \widehat{\widehat{O}}_{c}\right)$ implies that all $f_{w^{\prime}}$ are zero.

Assume that there exists a relation as above with not all $f_{w^{\prime}}$ equal to zero. Let $l_{0}$ be the maximum length of an element such that $f_{w^{\prime}} \neq 0$. From the definition (8.10) of $T_{w}^{c}$, we have that

$$
T_{w^{\prime}}^{c}=1_{c} \sum_{w^{\prime \prime} \in W_{0}} \gamma_{w^{\prime}, w^{\prime \prime}} T_{w^{\prime \prime}}
$$

where $\gamma_{w^{\prime}, w^{\prime \prime}} \in K$ are such that $\gamma_{w^{\prime}, w^{\prime \prime}}=0$ unless $w^{\prime \prime} \leq w^{\prime}$ in the Bruhat order and $\gamma_{w^{\prime}, w^{\prime}} \neq 0$. Hence, from (c) it follows that

(d) $\sum_{w^{\prime} \in W_{0}: l\left(w^{\prime}\right) \leq l_{0}} \tilde{f}_{w^{\prime}} T_{w^{\prime}}=0$ (in $\widehat{H}_{F}$ ), where $\tilde{f}_{w^{\prime}} \in \widehat{K}$ are such that $\tilde{f}_{w^{\prime}}=f_{w^{\prime}} \cdot \gamma_{w^{\prime}, w^{\prime}}$ for $l\left(w^{\prime}\right)=l_{0}$.

The product in $\widehat{K}$ of a nonzero element of $\hat{\mathscr{O}}_{c}$ with a nonzero element of $K$ is nonzero, since the natural homomorphism $\hat{\mathscr{O}}_{\rightarrow} \hat{\mathscr{O}}_{t}$ is injective and $\hat{\mathscr{O}}_{t}$ is an integral domain for any $t \in c$. It follows that $\tilde{f}_{w^{\prime}} \neq 0$ for some $w^{\prime}$ with $l\left(w^{\prime}\right)=l_{0}$ and, therefore, (d) contradicts 7.4(a). This contradiction proves (c). The proposition is proved.

\subsection{Proof of Theorem 8.6. Recall that $c \in \mathscr{P}$ is fixed.}

For any $c^{\prime} \in \mathscr{P}$, we choose a sequence $\zeta\left(c^{\prime}\right)=\left\{\alpha_{1}, \alpha_{2}, \ldots, \alpha_{p}\right\}$ in $\Pi$ such that $c \neq s_{\alpha_{p}}(c) \neq s_{\alpha_{p-1}} s_{\alpha_{p}}(c) \neq \cdots \neq s_{\alpha_{1}} s_{\alpha_{2}} \cdots s_{\alpha_{p}}(c)=c^{\prime}$.

For any $c^{\prime}, c^{\prime \prime} \in \mathscr{P}$, we define $\Delta_{c^{\prime}, c^{\prime \prime}}:{ }_{c} \widehat{H}_{c} \rightarrow{ }_{c^{\prime}} \widehat{H}_{c^{\prime \prime}}$ by

$$
\begin{aligned}
\Delta_{c^{\prime}, c^{\prime \prime}}(h) & =\tau^{\alpha_{1}} \tau^{\alpha_{2}} \cdots \tau^{\alpha_{p}} h \tau^{\beta_{p^{\prime}}} \tau^{\beta_{p^{\prime}-1}} \cdots \tau^{\beta_{1}} \\
& =\left(T_{s_{\alpha_{1}}}^{c^{\prime}} T_{s_{\alpha_{2}}}^{s_{a_{1}}\left(c^{\prime}\right)} \cdots\right) h\left(T_{s_{\beta_{p^{\prime}}}}^{c} T_{\beta_{p^{\prime}-1}}^{s_{\beta^{\prime}}(c)} \cdots\right),
\end{aligned}
$$

where $\zeta\left(c^{\prime}\right)=\left\{\alpha_{1}, \alpha_{2}, \ldots, \alpha_{p}\right\}, \zeta\left(c^{\prime \prime}\right)=\left\{\beta_{1}, \beta_{2}, \ldots, \beta_{p^{\prime}}\right\}$. 
$\Delta_{c^{\prime}, c^{\prime \prime}}$ is an isomorphism since $\tau^{\alpha} \tau^{\alpha}=1$ for $\alpha \in \Pi$.

We have clearly for $h, h_{1} \in{ }_{c} \widehat{H}_{c}$,

$$
\Delta_{c^{\prime}, c^{\prime \prime}}(h) \Delta_{c_{1}^{\prime}, c_{1}^{\prime \prime}}\left(h_{1}\right)= \begin{cases}\Delta_{c^{\prime}, c_{1}^{\prime \prime}}\left(h h^{\prime}\right), & \text { if } c^{\prime \prime}=c_{1}^{\prime}, \\ 0, & \text { if } c^{\prime \prime} \neq c_{1}^{\prime} .\end{cases}
$$

Hence, the map which associates to any square matrix $\left(h_{c^{\prime}, c^{\prime \prime}}\right)$ with entries in ${ }_{c} \widehat{H}_{c}$, indexed by $\left(c^{\prime}, c^{\prime \prime}\right) \in \mathscr{P} \times \mathscr{P}$, the element $\sum_{\left(c^{\prime}, c^{\prime \prime}\right) \in \mathscr{P} \times \mathscr{P}} \Delta_{c^{\prime}, c^{\prime \prime}}\left(h_{c^{\prime}, c^{\prime \prime}}\right) \in \widehat{H}$ defines a ring isomorphism

(a) $\left(\widehat{H}_{c}\right)_{n} \stackrel{\approx}{\rightrightarrows} \widehat{H}($ see $8.7(\mathrm{e}))$.

Here $\left({ }_{c} \widehat{H}_{c}\right)_{n}$ is the ring of $n \times n$ matrices $(n=\# \mathscr{P})$ with entries in the ring ${ }_{c} \widehat{H}_{c}$. By 7.4(b) for $\widehat{H}(c)$ instead of $\widehat{H}$, we have $\widehat{H}(c)=\bigoplus_{w \in W_{0}^{c}} \widehat{\mathscr{O}}_{c} T_{w, c}\left(T_{w, c}\right.$ is as in 8.3).

By definition of $\widehat{H}_{c}[\Gamma(c)]$, we have

$$
\widehat{H}_{c}[\Gamma(c)]=\bigoplus_{\substack{w \in W_{0}^{c} \\ \gamma \in \Gamma(c)}} \hat{\mathscr{O}}_{c} T_{w, c} \gamma .
$$

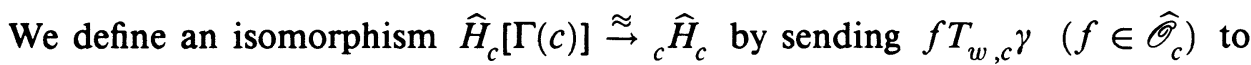
$f T_{w}^{c} T_{\gamma}^{c}$. From Lemma 8.13(c) and (d) and Lemma 8.14, we see that this is compatible with the ring structures. Combining this with (a), we get a ring isomorphism $\widehat{H}_{c}[\Gamma(c)]_{n} \cong \widehat{H}$. From the definitions, it follows easily that this is an isomorphism of $\hat{\mathscr{O}}^{W_{0}}$-algebras.

8.17. Remark. The isomorphism we have constructed depends on the choice of sequences $\zeta\left(c^{\prime}\right)$. However, another choice will only change the isomorphism into its composition with an inner automorphism of $\widehat{H}(c)[\Gamma(c)]_{n}$ defined by an $n \times n$ diagonal invertible matrix.

\section{SECOND REDUCTION THEOREM}

9.1. We preserve the setup of $\S 3$. Assume that we are given a $W_{0}$-orbit $\Sigma$ in $\mathscr{T}$ and an element $v_{0} \in \mathbf{C}^{*}$ of infinite order. Let $\left\langle v_{0}\right\rangle$ be as in 8.1.

We make the following assumption:

(a) if $t \in \Sigma$ and $\alpha \in R$, then

$$
\begin{cases}\theta_{\alpha}(t) \in\left\langle v_{0}\right\rangle, & \text { if } \check{\alpha} \notin 2 Y, \\ \theta_{\alpha}(t) \in \pm\left\langle v_{0}\right\rangle, & \text { if } \check{\alpha} \in 2 Y .\end{cases}
$$

9.2. The exponential map $e: \mathbf{C} \rightarrow \mathbf{C}^{*}\left(z \rightarrow e(z)=e^{z}\right)$ induces a homomorphism of complex Lie groups $\mathfrak{t}=Y \otimes \mathbf{C} \stackrel{1 \otimes e}{\rightarrow} Y \otimes \mathbf{C}^{*}=\mathscr{T}$ which will be denoted e. It is $W_{0}$-equivariant.

We select $r_{0} \in \mathbf{C}$ such that $v_{0}=e^{r_{0}}$.

We define a map $\Sigma \rightarrow t$ as follows. 
Let $t \in \Sigma$. For any $\alpha \in R$, let $n_{\alpha} \in \mathbf{Z}$ be such that $\theta_{\alpha}(t)= \pm v_{0}^{n_{\alpha}}$ (see 9.1(a)). Since $v_{0}$ is of infinite order, we have

$$
\left\{\begin{array}{l}
n_{\alpha+\beta}=n_{\alpha}+n_{\beta}, \quad \text { whenever } \alpha, \beta, \alpha+\beta \in R \\
n_{\alpha}+n_{-\alpha}=0, \quad \text { for all } \alpha \in R
\end{array}\right.
$$

Hence, there is a unique element $\bar{t}$ in the C-subspace of $\mathfrak{t}$ generated by $\check{R}$ such that $\alpha(\bar{t})=n_{\alpha} r_{0}$ for all $\alpha \in R$.

Then $t \mapsto \bar{t}$ is the required map $\Sigma \rightarrow \mathfrak{t}$. This map is clearly $W_{0}$-equivariant. Hence, its image $\bar{\Sigma}$ is a $W_{0}$-orbit in $\mathfrak{t}$.

We now define for any $t \in \Sigma$ an element $t_{0} \in \mathscr{T}$ by $t_{0}=t \cdot \mathfrak{e}(\bar{t})^{-1}$. The map $\Sigma \rightarrow \mathscr{T}, t \mapsto t_{0}$ is clearly $W_{0}$-equivariant. On the other hand, for each $t \in \Sigma$,

(a) $t_{0}$ is $W_{0}$-invariant

(see below). Hence, $t_{0}$ is necessarily independent of the choice $t$ (it depends on $\Sigma$ ). Let us prove (a). From the definitions, we have for any $\alpha \in R$,

$$
\theta_{\alpha}\left(t_{0}\right)=\theta_{\alpha}(t) \theta_{\alpha}(\mathfrak{e}(\bar{t}))^{-1}=\theta_{\alpha}(t) v_{0}^{-n_{\alpha}}= \begin{cases}1, & \text { if } \check{\alpha} \notin 2 Y, \\ \pm 1, & \text { if } \check{\alpha} \in 2 Y,\end{cases}
$$

so that (a) follows from Lemma 3.15.

We can now define

(b) $\bar{\Sigma} \rightarrow \Sigma\left(\bar{t} \mapsto t_{0} \mathfrak{e}(\bar{t})\right)$,

where $t_{0}$ is defined as above in terms of any $t \in \Sigma$. Then

(c) the map (b) is a $W_{0}$-equivariant bijection.

(Its inverse is $t \mapsto \bar{t}$.)

We shall use the notation $(\bar{H}, \overline{\mathscr{O}}, \ldots)$ and results of $\S \S 4$ and 5 for the particular $t_{0} \in \mathscr{T}$ considered above. We shall also use the notation and results of $\S 7$ relative to $\Sigma$ and $\bar{\Sigma}$ as above. We can state the following result.

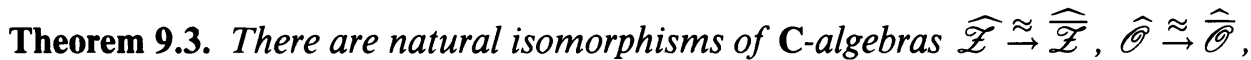
$\widehat{H} \approx \widehat{\rightrightarrows} \hat{H}$. Moreover, the last two isomorphisms are compatible with the $\widehat{\mathscr{Z}}$-and $\widehat{\widehat{\mathcal{X}}}$-algebra structures, via the first isomorphism.

9.4. For the proof, we shall need a lemma. We fix $\alpha \in \Pi$, and we regard $\mathscr{G}(\alpha)$ (resp. $g(\alpha))$ as a meromorphic function on $\mathscr{T} \times \mathbf{C}^{*}($ resp. $\mathfrak{t} \times \mathbf{C})($ see 3.8 and Proposition 4.4). Composing $\mathscr{G}(\alpha)$ with the holomorphic map $\psi: \mathfrak{t} \times \mathbf{C} \rightarrow$ $\mathscr{T} \times \mathbf{C}^{*},(\xi, z) \mapsto\left(t_{0} \mathfrak{e}(\xi), e^{z}\right)$, we obtain a meromorphic function $\tilde{\mathscr{G}}(\alpha)$ on $\mathfrak{t} \times \mathbf{C}$.

Lemma 9.5. The meromorphic function $\tilde{\mathscr{G}}(\alpha) g(\alpha)^{-1}$ is holomorphic and nonvanishing at all points of $\bar{\Sigma} \times\left\{r_{0}\right\}$. 
Proof. We have for $(\xi, z) \in \mathfrak{t} \times \mathbf{C}$,

$$
\begin{aligned}
& \left(\tilde{\mathscr{G}}(\alpha) g(\alpha)^{-1}\right)(\xi, z) \\
& \quad= \begin{cases}\frac{e^{\alpha(\xi)+2 \lambda(\alpha) z}-1}{\alpha(\xi)+2 \lambda(\alpha) z} \cdot \frac{\alpha(\xi)}{e^{\alpha(\xi)}-1}, \quad \text { if } \check{\alpha} \notin 2 Y, \\
\frac{\left(\theta_{\alpha}\left(t_{0}\right) e^{\alpha(\xi)+\left(\lambda(\alpha)+\lambda^{*}(\alpha)\right) z}-1\right)\left(\theta_{\alpha}\left(t_{0}\right) e^{\alpha(\xi)+\left(\lambda(\alpha)-\lambda^{*}(\alpha)\right) z}+1\right)}{\alpha(\xi)+\left(\lambda(\alpha)+\theta_{\alpha}\left(t_{0}\right) \lambda^{*}(\alpha)\right) z} & \frac{\alpha(\xi)}{e^{2 \alpha(\xi)}-1}, \\
& \text { if } \check{\alpha} \in 2 Y .\end{cases}
\end{aligned}
$$

Recall that $\theta_{\alpha}\left(t_{0}\right)= \pm 1$ if $\check{\alpha} \in 2 Y$. Hence, in that case we can also write

$$
\begin{aligned}
& \left(\tilde{\mathscr{G}}(\alpha) g(\alpha)^{-1}\right)(\xi, z) \\
& \quad=\frac{e^{\alpha(\xi)+\left(\lambda(\alpha)+\theta_{\alpha}\left(t_{0}\right) \lambda^{*}(\alpha)\right)}-1}{\alpha(\xi)+\left(\lambda(\alpha)+\theta_{\alpha}\left(t_{0}\right) \lambda^{*}(\alpha)\right) z} \cdot\left(e^{\alpha(\xi)+\left(\lambda(\alpha)-\theta_{\alpha}\left(t_{0}\right) \lambda^{*}(\alpha)\right) z}+1\right) \frac{\alpha(\xi)}{e^{2 \alpha(\xi)}-1} .
\end{aligned}
$$

To show holomorphicity, it is enough to show that for all $\bar{t} \in \bar{\Sigma}$

(a)

$$
\alpha(\bar{t}) \notin \begin{cases}2 \pi i \mathbf{Z}-\{0\}, & \text { if } \check{\alpha} \notin 2 Y, \\ \pi i \mathbf{Z}-\{0\}, & \text { if } \check{\alpha} \in 2 Y .\end{cases}
$$

To show nonvanishing, it is enough to show that for all $\bar{t} \in \bar{\Sigma}$

(b)

$$
\begin{aligned}
& \alpha(\bar{t})+2 \lambda(\alpha) r_{0} \notin 2 \pi i \mathbf{Z}-\{0\}, \quad \text { if } \check{\alpha} \notin 2 Y \text {, } \\
& \left.\begin{array}{c}
\alpha(\bar{t})+\left(\lambda(\alpha)+\theta_{\alpha}\left(t_{0}\right) \lambda^{*}(\alpha)\right) r_{0} \notin 2 \pi i \mathbf{Z}-\{0\} \\
\alpha(\bar{t})+\left(\lambda(\alpha)-\theta_{\alpha}\left(t_{0}\right) \lambda^{*}(\alpha)\right) r_{0} \notin 2 \pi i\left(\mathbf{Z}+\frac{1}{2}\right)
\end{array}\right\}, \quad \text { if } \check{\alpha} \in 2 Y .
\end{aligned}
$$

Substituting $\alpha(\bar{t})=n r_{0} \quad(n \in \mathbf{Z})$, we see that if one of the statements (a) or (b) is violated, we would have $r_{0}=2 \pi i n^{\prime} / n^{\prime \prime}$ for some nonzero integers $n^{\prime}, n^{\prime \prime}$. Hence, $v_{0}=e^{r_{0}}$ would be a root of 1 , contradicting our assumptions in 9.1.

9.6. Proof of Theorem 9.3. Since $\psi$ in 9.4 is locally a holomorphic isomorphism, it defines for each $\bar{t} \in \bar{\Sigma}$ an isomorphism

$$
\hat{\mathscr{O}}_{t, v_{0}}^{a n} \underset{\sim}{\approx} \hat{\mathscr{O}}_{\bar{t}, r_{0}}^{a n} \text {, }
$$

where $t=t_{0} \mathfrak{e}(\bar{t})$. Taking the direct sum over all $\bar{t} \in \bar{\Sigma}$ and using 9.2(c) we get an isomorphism $\bigoplus_{t \in \Sigma} \hat{\mathscr{O}}_{t, v_{0}}^{a n} \rightarrow \bigoplus_{\bar{t} \in \bar{\Sigma}} \hat{\overline{\mathscr{O}}}_{\bar{t}, r_{0}}^{a n}$. Using 7.6(a) and 7.5(a) and (b), this can be regarded as an isomorphism $\hat{\mathscr{O}} \approx \hat{\rightarrow} \hat{\mathscr{O}}$. This is clearly compatible with the $W_{0}$-actions. Taking $W_{0}$-invariants, we get an isomorphism $\widehat{\mathscr{Z}} \cong \widehat{\rightrightarrows} \widehat{\mathscr{Z}}$. We also get an isomorphism $j: \bar{K} \approx \widehat{\approx} \hat{\bar{K}}$ of the full rings of quotients of $\widehat{\mathscr{O}}, \hat{\mathscr{O}}$, which is again $W_{0}$-equivariant. We define $j^{\prime}: \widehat{H}_{F} \rightarrow \widehat{\bar{H}}_{\bar{F}}$ by

$$
j^{\prime}\left(\sum_{w} k_{w} \tau_{w}\right)=\sum_{w} j\left(k_{w}\right) \bar{\tau}_{w} \quad\left(k_{w} \in \widehat{K}\right)(\text { see } 7.4(\mathrm{a})) .
$$


This is compatible with multiplication:

$$
\begin{aligned}
j^{\prime}\left(k_{w} \tau_{w} \cdot k_{w^{\prime}} \tau_{w^{\prime}}\right) & =j^{\prime}\left(k_{w} w\left(k_{w^{\prime}}\right) \tau_{w} \tau_{w^{\prime}}\right)=j^{\prime}\left(k_{w} w\left(k_{w^{\prime}}\right) \tau_{w w^{\prime}}\right) \\
& =j\left(k_{w} w\left(k_{w^{\prime}}\right)\right) \bar{\tau}_{w w^{\prime}}=j\left(k_{w}\right) w\left(j\left(k_{w^{\prime}}\right)\right) \bar{\tau}_{w^{\prime}} \bar{\tau}_{w^{\prime}} \\
& =j\left(k_{w}\right) \bar{\tau}_{w} i\left(k_{w^{\prime}}\right) \bar{\tau}_{w^{\prime}}=j^{\prime}\left(k_{w} \tau_{w}\right) j^{\prime}\left(k_{w^{\prime}} \tau_{w^{\prime}}\right) .
\end{aligned}
$$

(We have used Proposition 5.2.)

We will show that $j^{\prime}$ maps $\widehat{H}\left(\subset \widehat{H}_{F}\right)$ isomorphically onto $\widehat{\bar{H}}\left(\subset \widehat{\bar{H}}_{\bar{F}}\right)$.

From 7.4(b), we know that $\widehat{H}$ is the subring of $\widehat{H}_{F}$ generated by $T_{s_{\alpha}}+1$ $(\alpha \in \Pi)$ and by $\hat{\mathscr{O}}$ and that $\hat{\bar{H}}$ is the subring of $\hat{\bar{H}}_{F}$ generated by $t_{s_{\alpha}}+1$ $(\alpha \in \Pi)$ and by $\hat{\mathscr{O}}$. Moreover, by definition, $j^{\prime}$ defines an isomorphism $\hat{\mathscr{O}} \rightarrow \hat{\mathscr{O}}$ and

$$
\begin{aligned}
j^{\prime}\left(T_{s_{\alpha}}+1\right) & =j^{\prime}\left(\left(\tau^{\alpha}+1\right) \mathscr{G}(\alpha)\right) \\
& =\left(\bar{\tau}^{\alpha}+1\right) j(\mathscr{G}(\alpha)) \\
& =\left(t_{s_{\alpha}}+1\right) g(\alpha)^{-1} j(\mathscr{G}(\alpha)) .
\end{aligned}
$$

Hence, it is enough to show that $j(\mathscr{G}(\alpha)) g(\alpha)^{-1} \in \widehat{K}$ is an invertible element of $\hat{\mathscr{O}}$, for any $\alpha \in \Pi$. But this follows from Lemma 9.5. The theorem is proved.

9.7. In the previous results we have assumed (see 9.1) that $v_{0}$ is of infinite order.

Analogous results hold for $v_{0}=1$. In this case, if $\Sigma$ is a $W_{0}$-orbit in $\mathscr{T}$ satisfying $9.1(\mathrm{a})$, then by Lemma $3.15, \Sigma$ consists of a single ( $W_{0}$-invariant) element $t_{0}$. We define $r_{0}=0$ and $\bar{\Sigma}=\{0\} \subset \mathfrak{t}$. The bijection 9.2(b) continues to hold.

The proof of Lemma 9.5 applies without change (the left-hand sides of 9.5(a), (b) are all zero). Hence, the statement (and proof) of Theorem 9.3 continues to hold (without change).

\section{ON SIMPLE $H$-MODULES}

10.1. For any ring $A$, we denote by $\operatorname{Irr} A$ the set of isomorphism classes of simple $A$-modules. If $M$ is an $A$-module, then $M \oplus \cdots \oplus M$ ( $n$ copies) can be regarded in an obvious way as an $A_{n}$-module $\left(A_{n}\right.$ as in 8.5), and $M \rightarrow$ $M \oplus \cdots \oplus M$ defines a bijection

(a) $\operatorname{Irr} A \stackrel{\approx}{\rightarrow} \operatorname{Irr} A_{n}$.

10.2. We preserve the setup of $\S 3$. If $M$ is a simple $H$-module, then $\mathscr{Z}$ acts on $M$ by scalars (by a well-known version of Schur's lemma due to Dixmier). Hence, there is a unique maximal ideal $J_{\left(\Sigma, v_{0}\right)}$ of $\mathscr{Z}$ (see 7.2) such that $J_{\left(\Sigma, v_{0}\right)} M$ $=0$. This defines a partition

(a) $\operatorname{Irr} H=\bigsqcup_{\left(\Sigma, v_{0}\right)} \operatorname{Irr}_{\left(\Sigma, v_{0}\right)} H$,

where $\Sigma$ runs over all $W_{0}$-orbits in $\mathscr{T}$ and $v_{0}$ runs over $\mathbf{C}^{*} ; \operatorname{Irr}_{\left(\Sigma, v_{0}\right)} H$ consists of those $M$ for which $J_{\left(\Sigma, v_{0}\right)} M=0$. 
10.3. We now fix $\left(\Sigma, v_{0}\right)$ as above, and let $\widehat{\mathscr{Z}}, \widehat{H}$ be the corresponding completions of $\mathscr{Z}, H$ (see 7.3). We denote by $\widehat{J}_{\left(\Sigma, v_{0}\right)}$ the unique maximal ideal of $\widehat{\mathscr{Z}}$. For any $\widehat{\mathscr{Z}}$-algebra $A$, we denote by $\operatorname{Irr}_{0} A$ the set of all $M^{\prime} \in \operatorname{Irr} A$ for which $\widehat{J}_{\left(\Sigma, v_{0}\right)} M^{\prime}=0$. We have

$$
H / J_{\left(\Sigma, v_{0}\right)} H=\widehat{H} / \widehat{J}_{\left(\Sigma, v_{0}\right)} \widehat{H}
$$

(a finite-dimensional algebra over $\mathbf{C}$ ). This defines a bijection

(a) $\operatorname{Irr}_{\left(\Sigma, v_{0}\right)} H \stackrel{\approx}{\rightarrow} \operatorname{Irr}_{0} \widehat{H}$.

10.4. We now assume that $v_{0}$ is of infinite order in $\mathbf{C}^{*}$. We select $r_{0} \in \mathbf{C}$ such that $e^{r_{0}}=v_{0}$. We partition $\Sigma$ in equivalence classes (in terms of $v_{0}$ ) as in 8.1, and we select an equivalence class $c$. By Theorem 8.6, we have an isomorphism of $\widehat{\mathscr{Z} \text {-algebras }}$

(a) $\widehat{H} \cong \widehat{H}_{c}[\Gamma(c)]_{n} \quad(n=\# \mathscr{P}$, as in 8.1).

By definition of $c$, the hypothesis of $\S 9$ (see 9.1(a)) is satisfied if $H, W_{0}$, $\Sigma, v_{0}$ are replaced by $H_{c}, W_{0}^{c}, c, v_{0}$. Hence, the constructions of $\S 9$ are applicable. In particular, the construction in 9.2 applied to $c$ instead of $\Sigma$ provides us with an element $t_{0} \in \mathscr{T}^{W_{0}^{c}}$ and a $W_{0}^{c}$ orbit $\bar{c} \subset \mathfrak{t}$ (instead of $\bar{\Sigma}$ ) such that $\mathrm{e}: \bar{c} \underset{\rightarrow}{\rightarrow}$. We define the graded algebra $\bar{H}_{c}$ associated to $H_{c}$ and this $t_{0}$ as in $\S 4$. Let $\widehat{\mathscr{Z}}_{c}$ (resp. $\widehat{\bar{H}}_{\bar{c}}$ or $\widehat{\mathscr{\mathscr { O }}}_{c}$ ) be the completions of the center $\overline{\mathscr{X}}_{c}$ of $\bar{H}_{c}$ (resp. of $\bar{H}_{c}^{c}$ itself or $\frac{c}{\mathscr{O}}$ ) with respect to the maximal ideal $\bar{J}$ of $\overline{\mathscr{Z}}_{\bar{c}}$ determined by $\left(\bar{c}, r_{0}\right)$ (as in 7.3).

Similarly, let $\widehat{\mathscr{Z}}_{c}, \widehat{H}_{c}$ be the completions of $\mathscr{Z}_{c}, H_{c}$ with respect to the maximal ideal of $\mathscr{Z}_{c}$ determined by $\left(c, v_{0}\right)$.

By Theorem 9.3 , we may identify naturally $\widehat{\mathscr{Z}}_{c}=\widehat{\mathscr{X}}_{c}$, and we have a natural isomorphism

(b) $\widehat{H}_{c} \cong \widehat{\bar{H}}_{c}$ of $\widehat{\mathscr{Z}}_{c^{-}}$(or $\widehat{\mathscr{\mathcal { X }}}_{\bar{c}^{-}}$) algebras.

Now $\Gamma(c)$ acts on $H_{c}$, and this induces an action of $\Gamma(c)$ on $\widehat{H}_{c}$. Moreover, from the definition of $\Gamma(c)$ and of $t_{0}$, we see that $t_{0}$ is $\Gamma(c)$-invariant. Hence, the $\Gamma(c)$-action on $H_{c}$ induces a $\Gamma(c)$-action on the associated graded algebra $\bar{H}_{\bar{c}}$ and on $\widehat{\bar{H}}_{\bar{c}}$.

Now (b) is compatible with the $\Gamma(c)$-actions (using the definitions). Hence, it extends to an isomorphism

(c) $\widehat{H}_{c}[\Gamma(c)] \cong \widehat{\bar{H}}_{c}[\Gamma(c)]$

taking $1 \cdot \gamma$ to $1 \cdot \gamma$ for $\gamma \in \Gamma(c)$. Then (c) is an isomorphism of algebras over $\widehat{\overline{\mathscr{Z}}}_{c}^{\Gamma(c)}=\widehat{\mathscr{Z}}_{c}^{\Gamma(c)}=\widehat{\mathscr{Z}}$ (see 8.5).

To simplify notation, we denote by $H^{\prime}$ the $\mathrm{C}$-algebra $\bar{H}_{\tau}[\Gamma(c)]$. Let $\mathscr{Z}^{\prime}$ be the center of $H^{\prime}$. 
Lemma 10.5. We have $\mathscr{Z}^{\prime}=\overline{\mathscr{O}}^{W_{0}(c)}\left(W_{0}(c)\right.$ as in 8.1).

This is a result of the same type as Proposition 4.5. Its proof follows almost word for word the proof of $[5,6.5]$.

10.6. From 10.5, we see that the maximal ideals of $\mathscr{Z}^{\prime}$ are in 1-1 correspondence with the $W_{0}(c)$-orbits in $\mathbf{t} \times \mathbf{C}$. Now $\left(\bar{c}, r_{0}\right)$ is such a $W_{0}(c)$-orbit. Hence, it defines a maximal ideal $J^{\prime}$ of $\mathscr{Z}^{\prime}$.

Let $\widehat{Z}^{\prime}, \widehat{\mathscr{O}}^{\prime}, \widehat{H}^{\prime}$ denote the $J^{\prime}$-adic completions of the $\mathscr{Z}^{\prime}$-algebras $\mathscr{Z}^{\prime}$, $\overline{\mathscr{O}}, H^{\prime}$, and let $\widehat{J}^{\prime}$ be the unique maximal ideal of $\widehat{\mathscr{Z}}$.

We have as in $7.5(\mathrm{~b}), \widehat{\overline{\mathscr{O}}}_{\bar{c}} \cong \bigoplus_{\bar{t} \in \bar{c}} \widehat{\widehat{\mathscr{O}}}_{\bar{t}}$ and similarly, $\hat{\overline{\mathscr{O}}}^{\prime}=\bigoplus_{i \in \bar{c}} \hat{\mathscr{O}}_{\bar{t}}$. Hence,

(a) $\hat{\overline{\mathscr{O}}}^{\prime}=\hat{\overline{\mathscr{O}}}_{\bar{c}}$.

Taking $W_{0}(c)$-invariants in (a), we obtain

(b) $\widehat{\mathscr{Z}}^{\prime}=\left(\widehat{\overline{\mathscr{Z}}}_{\bar{c}}\right)^{\Gamma_{0}(c)}$.

As we have seen in 10.4 , we have $\left(\widehat{\mathscr{Z}}_{c}\right)^{\Gamma_{0}(c)}=\left(\widehat{\mathscr{Z}}_{c}\right)^{\Gamma(c)}=\widehat{\mathscr{Z}}$. Hence, from (b) we deduce

(c) $\widehat{\mathscr{Z}}^{\prime}=\widehat{\mathscr{Z}}$.

In particular, $\widehat{J}^{\prime}=\widehat{J}_{\left(\Sigma, v_{0}\right)}$.

Now (a) shows that the $\bar{J}$-adic and $J^{\prime}$-adic completions of $\overline{\mathscr{O}}$ coincide. Since $\bar{H}_{\bar{c}}$ is a free left $\overline{\mathscr{O}}$-module, it follows that the $\bar{J}$-adic and $J^{\prime}$-adic completions of $\bar{H}_{\bar{c}}$ coincide.

It also follows that

(d) $\widehat{H}^{\prime}=\widehat{\bar{H}}_{c}[\Gamma(c)]$.

Let $\operatorname{Irr}_{\left(\bar{c}, r_{0}\right)} H^{\prime}$ be the set of all $M^{\prime} \in \operatorname{Irr} H^{\prime}$ such that $J^{\prime} M^{\prime}=0$. We have

$$
H^{\prime} / J^{\prime} H^{\prime}=\widehat{H}^{\prime} / \widehat{J}^{\prime} H^{\prime}
$$

(a finite-dimensional algebra over $\mathbf{C}$ ). Hence, we have a natural bijection

(e) $\operatorname{Irr}_{\left(\bar{c}, r_{0}\right)} H^{\prime} \approx \operatorname{Irr}_{0} \widehat{H}^{\prime}$.

10.7. From $10.4(\mathrm{c}), 10.6(\mathrm{~d})$ and (e), we obtain a natural bijection

$$
\operatorname{Irr}_{0} \widehat{H}_{c}[\Gamma(c)] \cong \operatorname{Irr}_{\left(\bar{c}, r_{0}\right)} H^{\prime} \text {. }
$$

Using 10.1(a), this gives rise to a bijection

$$
\operatorname{Irr}_{0} \widehat{H}_{c}[\Gamma(c)]_{n} \cong \operatorname{Irr}_{\left(\bar{c}, r_{0}\right)} H^{\prime} .
$$

Combining this with 10.4 , we obtain a bijection

$$
\operatorname{Irr}_{0} \widehat{H} \cong \operatorname{Irr}_{\left(\bar{c}, r_{0}\right)} H^{\prime} .
$$

Using this and 10.3(a), we obtain the main result of this paper.

Corollary 10.8. Recall that $v_{0}$ is assumed to have infinite order. There is a natural bijection

$$
\operatorname{Irr}_{\left(\Sigma, v_{0}\right)} H \cong \operatorname{Irr}_{\left(\bar{c}, r_{0}\right)} \bar{H}_{c}[\Gamma(c)]
$$


10.9. The same proof provides (for $v_{0}$ of infinite order) an equivalence of categories between $\operatorname{Mod}_{\left(\Sigma, v_{0}\right)} H$ (the category of $H$-modules of finite dimension over C, annihilated by some power of $J_{\left(\Sigma, v_{0}\right)}$ ) and $\operatorname{Mod}_{\left(\bar{c}, r_{0}\right)} \bar{H}_{\bar{c}}[\Gamma(c)]$ (the category of $\bar{H}_{\tau}[\Gamma(c)]$-modules of finite dimension over $\mathbf{C}$, annihilated by some power of $J^{\prime}$ ). The dimension of the module in the first category is \# $\operatorname{dim} \mathscr{P}$ times the dimension of the corresponding module in the second category.

This remains true when $v_{0}=1$ (we then take $r_{0}=0$ ), see 9.7. In this case, we have $\bar{c}=\{0\}, \Gamma(c)=\{1\}$.

\section{REFERENCES}

1. D. Kazhdan and G. Lusztig, Proof of the Deligne-Langlands conjecture for Hecke algebras, Invent. Math. 87 (1987), 153-215.

2. R. Kilmoyer, Principal series representations of finite Chevalley groups, J. Algebra 51 (1978), 300-319.

3. G. Lusztig, Singularities, character formulas and a q-analog of weight multiplicities, Astérisque 101-102 (1983), 208-229.

4. _ Some examples of square integrable representations of semisimple p-adic groups, Trans. Amer. Math. Soc. 277 (1983), 623-653.

5. __ Cuspidal local systems and graded Hecke algebras I, Inst. Hautes Études Sci. Publ. Math. 67 (1988), 145-202.

6. I. G. Macdonald, Spherical functions on a group of p-adic type, Publ. Ramanujan Inst., no. 2, Madras, 1971.

Department of Mathematics, Massachusetts institute of Technology, Cambridge, MASSACHUSETTS 02139 